(C) 2016 IEEE. Personal use of this material is permitted. Permission from IEEE must be obtained for all other uses, in any current or future media, including reprinting/republishing this material for advertising or promotional purposes, creating new collective works, for resale or redistribution to servers or lists, or reuse of any copyrighted component of this work in other works. 


\title{
A Signal-space Aligned Network Coding Approach to Distributed MIMO
}

\author{
Tao Yang, Member, IEEE, Xiaojun Yuan, Member, IEEE, Qifu Tyler Sun, Member, IEEE
}

\begin{abstract}
This paper studies an uplink distributed MIMO (DMIMO) system that consists of $K$ users and $K^{\prime}$ distributed base stations (BSs), where the BSs are connected to a central unit (CU) via independent rate-constrained backhaul (BH) links. We propose a new signal-space aligned network coding scheme. First, a network coding generator matrix is selected subject to certain structural properties. Next, distributed linear precoding is employed by the users to create aligned signal-spaces at the BSs, according to the pattern determined by the network coding generator matrix. For each aligned signal-space at a BS, physical-layer network coding is utilized to compute the corresponding network-coded (NC) messages, where the actual number of $\mathrm{NC}$ messages forwarded to the $C U$ is determined by the $B H$ rate-constraint. We derive an achievable rate of the proposed scheme based on the existence of the NC generator matrix and signal-space alignment precoding matrices. For DMIMO with two and three BSs, the achievable rates and degrees of freedom (DoF) are evaluated and shown to outperform existing schemes. For example, for DMIMO with two BSs where each user and BS have $N$ and $N^{\prime}$ antennas, respectively, the proposed scheme achieves a DoF of $2 \min \left(N, N^{\prime}\right)-1$, if the $\mathbf{B H}$ capacity scales like $\left(2 \min \left(N, N^{\prime}\right)-1\right) \log S N R$. This leads to greater DoF compared to that utilizes the strategy for interference channel, whose DoF is $\min \left(N, N^{\prime}\right)$. Numerical results demonstrate the performance advantage of the proposed scheme.
\end{abstract}

Index Terms-Cloud RAN, CoMP, distributed MIMO, physicallayer network coding, compute-and-forward, interference alignment

\section{INTRODUCTION}

Infrastructure networks are widely considered for providing high data-rate broadband services, so as to meet the skyrocketing demands for data hungry applications. A major impediment to the successful implementation of such networks is interference. In traditional network design, interference mitigation is employed, e.g., by assigning orthogonal spectral resources to adjacent cells. As inspired by the advances in multiple-input multiple-output (MIMO) technology, distributed MIMO (DMIMO) systems have been intensively investigated. By mimicking a MIMO system using base station cooperation and multi-cell joint processing, D-MIMO can better deal with interference, thus enabling high frequency reuse factors and increased spectral efficiency. DMIMO finds applications in a wide range of current and future wireless networks, such as coordinated multi-point (CoMP) [1] in LTEadvanced and 5G cellular networks, local area networks with WiFi coordination, and mobile over Fiber to the $\mathrm{x}$ (FTTx) networks based on a multi-source multi-relay model [2].

In a DMIMO system, a number of mobile users are served by a number of distributed base stations (BSs). These separated BSs are connected to a central unit (CU) via independent backhaul links. Such a DMIMO system can also be regarded as a subclass of distributed-input distributed-output (DIDO) system [3], or cloud radio access network (C-RAN) [4] [5] [6] [7]. In most practical DMIMO scenarios, the backhaul links have limited capacity, which has tremendous impacts on the design of the networks [8] [9] [10]. This paper is concerned with the design of an uplink DMIMO system under practical backhaul rate constraints. The uplink DMIMO can be thought of as a MIMO interference channel with an additional second-hop connection to a central processing unit. It can also be viewed as a virtual MIMO multiple-access channel between the users and the $\mathrm{CU}$, with the BSs acting as relays.

\section{A. State of the Art}

The research in DMIMO with rate-constrained backhaul has attracted significant research efforts. The achievable rates and the relay strategies of the uplink DMIMO have been studied under a Wyner model [11] where decode-and-forward and compressand-forward were considered. Several recent works such as [12] and [13] attempts to characterize the information theoretical capacity. An approach based on quantize re-map and forward (QMF) was proposed in [12]. This approach was extended to a general network, referred to as noisy network coding [13]. In the QMF approach, the base stations perform vector quantization of their received signals at some rate satisfying the backhaul rate-constraint. It is shown that QMF or noisy network coding achieves a rate region within a bounded gap from the cut-set outer bound. Yet, from an implementation viewpoint, QMF and noisy network coding may require very high complexity due to vector quantization. The DMIMO systems have been studied from a CoMP perspective, where coordinated beamforming and joint processing are considered and compared [14] [15].

The compute-and-forward (CF) strategy can be employed in the uplink DMIMO system [16]. CF can be regarded as a theoretical notion and extension of physical-layer network coding (PNC), which harnesses interference through structured linear or lattice codes for more efficient communication over networks. In CF, each base station attempts to directly generate and forward some finite-field linear combinations of all users' messages, without decoding each individual messages. The major impairments of direct application of CF in DMIMO is the non-integer penalty and the high probability of rank deficiency [17]. Low complexity CF schemes and reverse CF have been studied in [18] for distributed multi-antenna systems.

For the case with global channel state information (CSI), the PNC or CF strategy can be better managed by incorporating 
signal-space alignment (SSA) techniques. For MIMO two-way relay channel, a generalized singular-value decomposition and an eigen-direction alignment precoding based PNC schemes were proposed, where the gap to the cut-set bound is almost closed [19]. The main idea is to create multiple signal-spaces where different users' signals are aligned. Each aligned signal-space provides a platform for PNC to compute one linear message combination. For a MIMO Y channel, where three users exchange information via a relay, the SSA based scheme was studied [20]. Considerable degrees of freedom gain was reported therein.

The SSA schemes extend the interference alignment (IA) for the interference channel [21]. In IA, the interference signals are placed into a "waste bin", while the desired signals can be decoded free from the interference in the waste bin. The structure of the interference in the bin is ignored, which is justified in the model of interference channel. Yet, for other network configurations, the SSA advances IA by exploiting both the structure of the desired signal and that of the interference to carry out PNC [19] [20]. An integer-forcing IA scheme was proposed where the PNC or CF mechanism was considered for interference channel [22]. To date, the impact of SSA and PNC on DMIMO with rate-constrained $\mathrm{BH}$ remains open in the literature.

\section{B. Contributions}

The main contributions of this paper are summarized as follows. We propose a new signal-space aligned network coding scheme for the DMIMO system with rate-constrained backhaul. First, a network coding generator matrix that satisfies certain structural properites is selected. This network coding generator matrix determines 1) the pattern of SSA and 2) the coefficients of the linear message-combinations (referred to as networkcoded (NC) messages) to be computed-and-forwarded by the BSs. Second, distributed linear precoding is designed and employed at the user nodes such that the signal-spaces are aligned at the BSs, according to the pre-determined pattern. Next, for each aligned signal-space at a BS, PNC (or CF) is utilized to directly compute the associated $\mathrm{NC}$ message. The $\mathrm{BH}$ rate constraint determines the actual number of NC messages forwarded to the CU. Finally, the CU recovers all users' messages by multiplying the inverse of the network coding generator matrix over the NC messages.

We derive an achievable rate of the proposed scheme. The proof is based on existence of network coding generator matrices, signal alignment precoding matrices and chain of nested lattice codes. For the parallel-relay and tri-relay networks, i.e., DMIMO with two and three BSs, the achievable rates and degrees of freedom (DoF) are explicitly evaluated. For example, for the parallel-relay network where each user has $N$ antennas and each BS has $N^{\prime}$ antennas, the proposed scheme can achieve a DoF of $2 \min \left(N, N^{\prime}\right)-1$, if the sum backhaul capacity scales like $\left(2 \min \left(N, N^{\prime}\right)-1\right) \log S N R$. This leads to significantly increased DoF compared to that utilizes the strategy for interference channel, whose DoF is given by $\min \left(N, N^{\prime}\right)$. Numerical results are also provided to demonstrate the performance advantage of the proposed scheme.

\section{Scopes}

This paper only considers signal-space alignment. No efforts will be made to investigate signal-scale alignment. Although the signal-scale alignment schemes, based on rational independence [23] [24], can be employed for obtaining DoF characterizations at extremely high SNR, it may offer little in terms of useful insights for the practical SNR values and finite precision channel knowledge, where the notion of rational independence loses its relevance [23] [24]. Signal-space alignment schemes, on the other hand, are desirable both for their analytical tractability as well as the useful insights they offer for practical SNR regime [25].

This paper considers constant (block-fading) channel rather than time-varying channel, and symbol-extension is not explored. It has been shown that alignment in spatial dimension through multiple antennas is more robust to practical limitations such as frequency offsets than alignment in time or frequency dimensions [25]. Although our proposed scheme can be extended to the case with symbol-extension for time-varying channel, the analysis and optimal design with symbol-extension is out of the scope of this paper and will be left to future works.

Throughout this paper, we shall use the following notations. A lower case non-bold character $a$ denotes a scalar. A lower case bold character a denotes a vector, which could be either a row vector or a column vector. The $\tau$ th entry of $\mathbf{a}$ is denoted by $a[\tau]$. The vector 2 -norm of $\mathbf{a}$ is denoted by $\|\mathbf{a}\|$. A vector $\widehat{\mathbf{a}}$ denotes a decision made (or an estimate) on the original vector a. An upper case bold character $\mathbf{A}$ denotes a matrix, and $\mathbf{a}_{i}$ denotes the $i$ th row or $i$ th column of $\mathbf{A}$. An upper case non-bold character with (or without) a "prime", i.e., $K^{\prime}$ (or $K$ ), is used to denote a parameter w.r.t. the BSs (or users). Denote $\max (a, 0)$ by $(a)^{+}$.

\section{SySTEM MODEL}

Consider a DMIMO system that consists of $K$ users and $K^{\prime}$ base stations (BSs), as shown in Fig. 1. User $i$ has $N_{i}$ antennas and BS $j$ has $N_{j}^{\prime}$ antennas, $i=1, \cdots, K, j=1, \cdots, K^{\prime}$. Without loss of generality, the users and the BSs are ordered such that $N_{1} \geq N_{2} \geq \cdots \geq N_{K}$ and $N_{1}^{\prime} \geq N_{2}^{\prime} \geq \cdots \geq N_{K^{\prime}}^{\prime}$. The BSs are connected to the $\mathrm{CU}$ via $K^{\prime}$ independent rate-constrained $\mathrm{BH}$ links. Note that the BSs are not mutually connected.

The DMIMO system consists of two layers: air-interface layer and backhaul $(\mathrm{BH})$ layer. In this paper, we consider the uplink scenario. At the air-interface layer, $K$ users transmit their messages towards the $K^{\prime}$ BSs through the air. At BH layer, each $\mathrm{BS}$ processes its received signal and forwards it to the $\mathrm{CU}$ via its BH. Upon collecting the signals from the $K^{\prime}$ BSs' $\mathrm{BH}$ links, the CU eventually recovers all $K$ users' messages.

\section{A. Air-Interface Layer}

User $i$ has a length- $m_{i}$ message sequence denoted by a row vector $\mathbf{b}_{i}, i \in\{1, \cdots, K\}$, where the entries of $\mathbf{b}_{i}$ belong to $\mathrm{GF}(q)$. User $i$ 's encoder maps $\mathbf{b}_{i}$ into a size $N_{i}$ by $n$ space-time signal matrix $\mathbf{X}_{i}$. Denote the encoding functions by

$$
\mathbf{X}_{i}=\varepsilon_{i}\left(\mathbf{b}_{i}\right), i=1,2, \cdots, K \text {. }
$$

The information rate of User $i$ is $R_{i}=\frac{m_{i}}{n} \log _{2} q$ bits per channeluse. Note that the encoder $\varepsilon_{i}(\cdot)$ incorporates the channel-code encoder, digital modulator and MIMO precoder. 


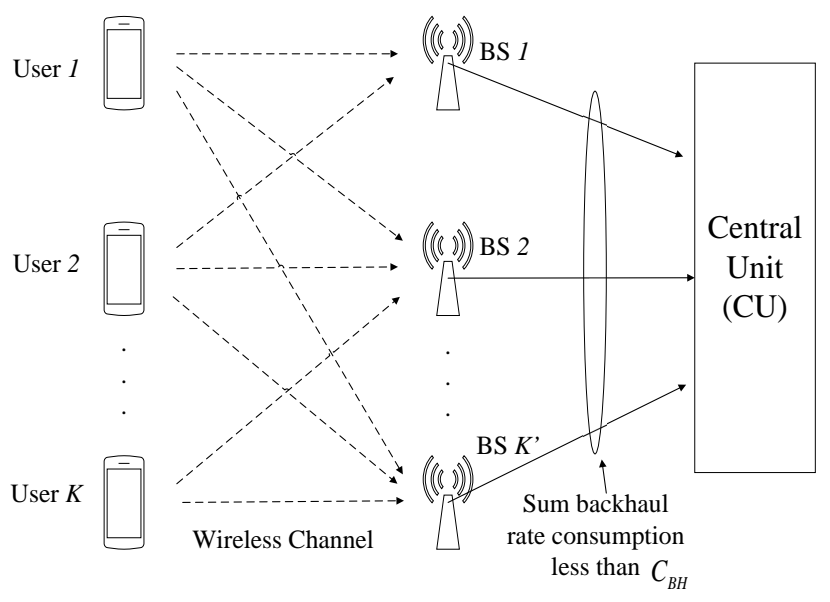

Fig. 1. Block diagram of an $K$-user $K^{\prime}$-BS uplink DMIMO system with rateconstrained backhaul.

We consider a block fading channel, i.e. the channel coefficients remain constant over a block of $n$ symbols and vary over blocks. In one block, the signal received by the $j$ th $\mathrm{BS}$ is

$$
\mathbf{Y}_{j}=\sum_{i=1}^{K} \mathbf{H}_{j, i} \mathbf{X}_{i}+\mathbf{Z}_{j}, j=1,2, \cdots, K^{\prime},
$$

where $\mathbf{H}_{j, i}$ denotes the channel coefficient matrix from User $i$ to BS $j$, and $\mathbf{Z}_{j}$ denotes the additive white Gaussian noise (AWGN) matrix with i.i.d. entries of zero mean and variance $\frac{N_{0}}{2}$ per realdimension. In this paper, global channel state information (CSI) and symbol synchronization is assumed.

Let $\mathbf{x}_{i}[t]$ be the $t$ th column of $\mathbf{X}_{i}$, denoting the signal vector transmitted by User $i$ 's multiple antennas at time instant $t, t=$ $1, \cdots, n$. A total power constraint is given by

$$
\sum_{i=1}^{K} E\left(\left|\mathbf{x}_{i}^{T}[t] \mathbf{x}_{i}[t]\right|^{2}\right) \leq E_{\text {total }} .
$$

The per-user signal-to-noise ratio (SNR) is defined as $\rho \triangleq \frac{E_{t o t a l}}{N_{0} K}$. In this paper, we only focus on the case with total power constraint. Our development can be extended to the case with individual power constraints.

\section{B. Backhaul Layer}

BS $j$ processes its received signal $\mathbf{Y}_{j}$. The resultant signal is generally written as $f_{j}\left(\mathbf{Y}_{j}\right), j=1, \cdots, K^{\prime}$., which is forwarded to the $\mathrm{CU}$ via the $j$ th $\mathrm{BH}$ link. As in [7], we consider a total $\mathrm{BH}$ rate constraint. ${ }^{1}$ Denote by $C_{B H}$ the maximum rate that can be supported by the $K^{\prime} \mathrm{BH}$ links in total for one block [26], [27] [28]. The rate-constraint under consideration is written as

$$
H\left(f_{1}\left(\mathbf{Y}_{1}\right), \cdots, f_{K^{\prime}}\left(\mathbf{Y}_{K^{\prime}}\right)\right) \leq C_{B H} .
$$

Here, $H($.$) stands for the entropy function. Error free transmis-$ sion of the signal over the BH links is assumed if (4) is met,

\footnotetext{
${ }^{1}$ Our proposed scheme also applies to individual backhaul rate constraints.
}

following from the distributed source coding theorem [29]. The normalized $\mathrm{BH}$ rate-constraint (per channel-use) is defined as

$$
\bar{C}_{B H}=\frac{C_{B H}}{n} .
$$

Let $\widehat{\mathbf{u}}_{j}=f_{j}\left(\mathbf{Y}_{j}\right), j=1,2, \cdots, K^{\prime}$. Upon collecting $\widehat{\mathbf{u}}_{1}, \cdots, \widehat{\mathbf{u}}_{j}$ from the BH links, the CU attempts to recover all $K$ users' messages $\mathbf{b}_{1}, \cdots, \mathbf{b}_{K}$. The decision made in the final decoding process is represented by

$$
\left[\widehat{\mathbf{b}}_{1}, \cdots, \widehat{\mathbf{b}}_{K}\right]=\operatorname{dec}\left(\widehat{\mathbf{u}}_{1}, \cdots, \widehat{\mathbf{u}}_{K^{\prime}}\right) .
$$

A decoding error is declared if $\widehat{\mathbf{b}}_{i} \neq \mathbf{b}_{i}$ for any $i \in\{1, \cdots, K\}$. Remark 1: By removing the second-hop (the BH layer) and letting each BS recover one specific user's message, the DMIMO system model becomes a $K$-user interference channel [21].

\section{Performance Metrics: Achievable Information Rate and De- grees of Freedom}

For given channel coefficient matrices $\mathbf{H}_{j, i}$, $i=1, \cdots, K, j=1, \cdots, K^{\prime}$, and given $\mathrm{BH}$ rate-constraints $C_{B H}$, a $\left(R_{1}, \cdots, R_{K}, n, P_{e}\right)$ code consists of the following:

1. $K$ encoding functions $\varepsilon_{1}(\cdot), \cdots, \varepsilon_{K}(\cdot)$ of rates $R_{1}, \cdots, R_{K}$ respectively.

2. $K^{\prime}$ functions $f_{1}(\cdot), \cdots, f_{K^{\prime}}(\cdot)$ at the BSs that satisfy the $\mathrm{BH}$ rate-constraint as specified in (4).

3. A decoding function $\operatorname{dec}(\cdot)$ at the $\mathrm{CU}$ as specified in (6).

4. An error probability $P_{e}$, given by

$$
P_{e}=\operatorname{Pr}\left\{\left[\widehat{\mathbf{b}}_{1}, \cdots, \widehat{\mathbf{b}}_{K}\right] \neq\left[\mathbf{b}_{1}, \cdots, \mathbf{b}_{K}\right]\right\} .
$$

A rate tuple $R_{1}, \cdots, R_{K}$ is said to be achievable if, for any $\epsilon>0$, there exists a $\left(R_{1}, \cdots, R_{K}, n, P_{e}\right)$ code such that $P_{e} \leq \epsilon$ as the block length $n \rightarrow \infty$.

A degrees of freedom (DoF) tuple $\left(d_{1}, \cdots, d_{K}\right)$ is said to be achievable if the achievable rate scales like

$$
R_{i} \propto d_{i} \log \rho
$$

for $i=1, \cdots, K$, as the SNR $\rho$ tends to infinity. An achievable total DoF is given by

$$
d_{\text {total }}=\sum_{i=1}^{K} d_{i}
$$

The objective is to investigate how to achieve a high rate or DoF for the DMIMO system presented above.

\section{Moderate Backhaul-Capacity Scenario}

The design of a DMIMO system under rate-constrained $\mathrm{BH}$ has received extensive interests [16], [26]. By and large, there are three categories w.r.t. the $\mathrm{BH}$ rate constraint. The most interested category is a "moderate-to-large BH" case, where the $\mathrm{BH}$ rateconstraint and information capacity of the air-interface are of the same order of magnitude. In such a scenario, the ratio between the $\mathrm{BH}$ rate and wireless rate could be from about one to a relatively number, e.g., the $\mathrm{BH}$ rate could be a few times greater than the capacity of the wireless channel. For a very "extremely rich $\mathrm{BH}^{\prime}$ " case, where the $\mathrm{BH}$ rate-constraint is of a much higher 
order than the air-interface capacity, the $\mathrm{BH}$ is sufficient for the BSs to simply forward their entire received signals to the CU. Then, the DMIMO reduces to a MIMO multiple-access channel with a common receiver of $\sum_{j=1}^{K^{\prime}} N_{j}^{\prime}$ co-located antennas. For a very "stringent $B H$ " case, where the $\mathrm{BH}$ rate-constraint is much smaller than the air-interface capacity, the throughput of the DMIMO is simply bottle-necked by the $\mathrm{BH}$ rate-constraint. This paper only focus on the moderate-to-large $\mathrm{BH}$ case, as the other two cases are of less theoretical and practical interests.

\section{Proposed Signal-Space Aligned Physical-Layer NeTwOrK CODING APPROACH}

Our proposed methodology consists of the following two key techniques:

1. Signal-space alignment precoding: Linear precoding matrices are designed for the $K$ transmitters such that the signal-spaces of the spatial streams of various users are desirably aligned at the BS receivers.

2. Physical-layer network coding (PNC): For each aligned signal-space, some linear combination of the users' messages is generated, following the notion of PNC or CF. The linear message-combinations, also referred to as the network-coded $(N C)$ message sequences, are forwarded to the $\mathrm{CU}$ via the $\mathrm{BH}$.

The $\mathrm{BH}$ rate-constraint limits the actual number of $\mathrm{NC}$ message sequences that are forwarded to the $\mathrm{CU}$. The proposed scheme is illustrated in Fig. 2.

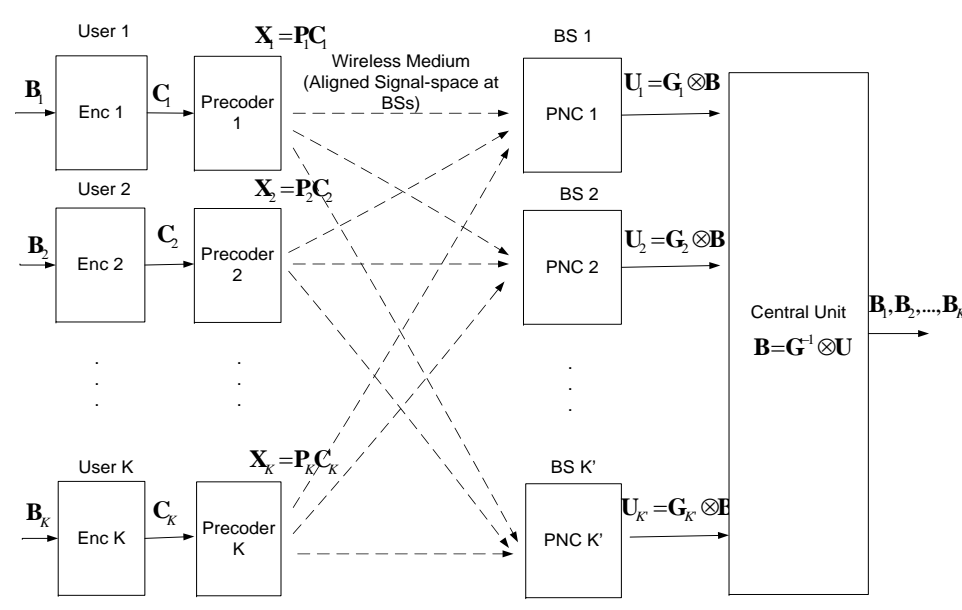

Fig. 2. Illustration of the proposed scheme. Each user encodes its message streams independently. Signal-space alignment precoding is then implemented over the streams. At the output of wireless channel, the signal spaces are aligned at the base stations according to the pattern given by G. Each user carries out PNC to compute the linear message combinations which are forwarded to the $\mathrm{CU}$, where the $\mathrm{BH}$ rate constraint determines the number of equations actually forwarded.

\section{A. Encoding}

1) Channel-coding and Modulation: User i's message sequence $\mathbf{b}_{i}, i=1, \cdots, K$, is truncated into $L_{i}$ segments of row vectors $\mathbf{b}_{i, 1}, \cdots, \mathbf{b}_{i, L_{i}}$, each represents an independent data stream. The lengths of the data streams are respectively denoted by $m_{i, 1}, \cdots, m_{i, L_{i}}$, where $\sum_{l=1}^{L_{i}} m_{i, l}=m_{i}$. The total number of data streams of all $K$ users is denoted by

$$
L=\sum_{i=1}^{K} L_{i}
$$

The data streams $\mathbf{b}_{i, 1}, \cdots, \mathbf{b}_{i, L_{i}}$ of User $i$ are independently coded and modulated, yielding sequences $\mathbf{c}_{i, 1}, \cdots, \mathbf{c}_{i, L_{i}}$ of a common length $n$. The coded-modulated sequences of User $i$ are collectively written as

$$
\mathbf{C}_{i}=\left[\begin{array}{c}
\mathbf{c}_{i, 1} \\
\vdots \\
\mathbf{c}_{i, L_{i}}
\end{array}\right]
$$

Note that the coding and modulation under consideration support PNC or $\mathrm{CF}$, which enables direct computation of linear combination of multiple users' messages without complete decoding of individual messages. From an information theoretic aspect, existence of nested lattice codes that are simultaneously "quantization-good" and "AWGN-good" are used to prove the results [30], [16]. From a practical aspect, non-binary low-density parity-check (LDPC) codes such as the random-coset irregular repeat-accumulate codes with iterative believe propagation decoding algorithm can be employed to support CF [31], [32].

2) Distributed Linear Precoding: Each user performs a linear precoding, yielding

$$
\mathbf{X}_{i}=\mathbf{P}_{i} \mathbf{C}_{i}, i=1, \cdots, K .
$$

Each row of $\mathbf{X}_{i}$ is referred to as a spatial stream. Each column of $\mathbf{P}_{i}$ specifies the signal-space of a spatial stream. Note that there are $L$ spatial streams in total for all users, each carries one data stream.

Consider that $\mathbf{C}_{i}$ is normalized, i.e., $E\left(\mathbf{C}_{i} \mathbf{C}_{i}^{H}\right)=\mathbf{I}$. Then, the power constraint in (3) becomes

$$
\sum_{i=1}^{K} \operatorname{Tr}\left(\mathbf{P}_{i} \mathbf{P}_{i}^{H}\right) \leq E_{\text {total }}
$$

The $K$ users transmit simultaneously. The BSs receive $\mathbf{Y}_{j}, j=$ $1, \cdots, K^{\prime}$, as specified in Eq. (2).

\section{B. Network Coding and Signal-space Alignment Precoding}

1) Preliminary on Network Coding: The message sequences of User $i$ is collectively written as

$$
\mathbf{B}_{i}=\left[\begin{array}{c}
\widetilde{\mathbf{b}}_{i, 1} \\
\vdots \\
\widetilde{\mathbf{b}}_{i, L_{i}}
\end{array}\right], i=1, \cdots, K,
$$

where $\widetilde{\mathbf{b}}_{i, l}$ is formed by padding $\mathbf{b}_{i, l}$ with $m_{\max }-m_{i, l}$ zeros, $m_{\max }=\max _{i, l} m_{i, l}$. The size of $\mathbf{B}_{i}$ is $L_{i}$ by $m_{\max }$. In addition, all message sequences are collectively denoted by a size $L$ by $m_{\max }$ matrix

$$
\mathbf{B}=\left[\mathbf{B}_{1}^{T}, \cdots, \mathbf{B}_{K}^{T}\right]^{T} .
$$


a) One Network-Coded (NC) Message Sequence: A linear combination of the users' message sequences, referred to as a $N C$ message sequence, is written as

$$
\mathbf{u}=\mathbf{g} \otimes \mathbf{B}
$$

where " $\otimes$ " denotes the multiplication in $\operatorname{GF}(q)$. Here, the length$L$ row vector $\mathrm{g}$ is referred to as a NC coefficient vector, whose $L$ entries specify the coefficients in the linear combination. The entries of $\mathrm{g}$ belong to $\mathrm{GF}(q)$.

b) Index Function: We define the following index function

$$
I(i, l) \triangleq \sum_{\gamma=1}^{i-1} L_{\gamma}+l .
$$

The $l$ th data stream of User $i$ is now located at the $I(i, l)$ th row of $\mathbf{B}$. The NC coefficient for the $l$ th spatial stream of User $i$ is specified by the $I(i, l)$-th entry of $\mathbf{g}$.

Let $\tau=I(i, l)$. The inverse of the index function is

$$
I^{-1}(\tau) \triangleq i \text { if } \sum_{\gamma=1}^{i-1} L_{\gamma}<\tau \leq \sum_{\gamma=1}^{i} L_{\gamma}
$$

The $\tau$ th row of $\mathbf{B}$ now belongs to User $I^{-1}(\tau)$. The $\tau$ th entry of $\mathbf{g}$ is exclusively related to User $I^{-1}(\tau)$. Note that the inverse index function can also return the index of the spatial stream $l$, which is omitted here for simplicity of presentation.

c) Multiple NC Message Sequences: Now consider that BS $j$ attempts to generate $L_{j}^{\prime} \mathrm{NC}$ message sequences $\mathbf{u}_{j, 1}, \cdots, \mathbf{u}_{j, L_{j}^{\prime}}$. These NC message sequences are collectively represented by

$$
\mathbf{U}_{j}=\left[\begin{array}{c}
\mathbf{u}_{j, 1} \\
\vdots \\
\mathbf{u}_{j, L_{j}^{\prime}}
\end{array}\right]=\left[\begin{array}{c}
\mathbf{g}_{j, 1} \otimes \mathbf{B} \\
\vdots \\
\mathbf{g}_{j, L_{j}^{\prime}} \otimes \mathbf{B}
\end{array}\right]
$$

where $\mathbf{g}_{j, l^{\prime}}$ denotes coefficient vector of the $l^{\prime}$ th NC message sequence, $l^{\prime}=1, \cdots, L_{j}^{\prime}$. The total number of NC message sequences of all BSs is $L^{\prime}=\sum_{j=1}^{K^{\prime}} L_{j}^{\prime}$. Without loss of generality, we assume $L_{1}^{\prime} \geq \cdots \geq L_{K^{\prime}}^{\prime}$, and $L_{1} \geq \cdots \geq L_{K}{ }^{2}$

d) NC Generator Matrix: Let

$$
\mathbf{G}_{j}=\left[\begin{array}{c}
\mathbf{g}_{j, 1} \\
\vdots \\
\mathbf{g}_{j, L_{j}^{\prime}}
\end{array}\right], j=1, \cdots, K^{\prime}
$$

whose rows collect the $L_{j}^{\prime} \mathrm{NC}$ coefficient vectors of BS $j$. Then Eq. (19) is written as

$$
\mathbf{U}_{j}=\mathbf{G}_{j} \otimes \mathbf{B} .
$$

Further, let

$$
\mathbf{G}=\left[\mathbf{G}_{1}^{T}, \cdots, \mathbf{G}_{K^{\prime}}^{T}\right]^{T}
$$

which collects all $L^{\prime} \mathrm{NC}$ coefficient vectors of all BSs. We refer to $\mathbf{G}$ as a $N C$ generator matrix.

\footnotetext{
${ }^{2}$ Recall that the BSs have been ordered such that $N_{1} \geq N_{2} \geq \cdots \geq N_{K^{\prime}}$. As such, for $j^{\prime}>j$, BS $j$ has a larger dimension in the received signal space than BS $j^{\prime}$, thus is likely to be able to generate more NC message sequences.
}

The $L^{\prime} \mathrm{NC}$ message sequences to be generated by all BSs can now be written as

$$
\mathbf{U}=\left[\mathbf{U}_{1}^{T}, \cdots, \mathbf{U}_{K^{\prime}}^{T}\right]^{T}=\mathbf{G} \otimes \mathbf{B} .
$$

Remark 2: The total number of NC message vectors generated by all BSs must be no less than the total number of spatial streams, i.e., $L^{\prime} \geq L$. In addition, the $\mathrm{NC}$ generator matrix $\mathbf{G}$ must have a full rank of $L$. These are necessary conditions for the $\mathrm{CU}$ to recover all messages, as will be seen later.

Remark 3: In this paper, we only focus on $L^{\prime}=L$, that is, the $\mathrm{NC}$ generator matrix $\mathbf{G}$ is square. This is because if $L^{\prime}>L$, at least one NC message sequence is linearly dependent on others, which may lead to a waste in the BH consumption.

Remark 4: The matrix $\mathbf{G}$ must be compatible to implementation of SSA and PNC. This imposes certain structural properties (Properties 1-3) as will be detailed in Section IV. A.

2) Signal-space Alignment (SSA) Precoding: The following notations will be used:

The columns of $\mathbf{P}_{i}$ are denoted by $\mathbf{p}_{i, l}, l=1, \cdots, L_{i}$. For convenience, the columns of $\mathbf{P}=\left[\mathbf{P}_{1}, \ldots, \mathbf{P}_{K}\right]$ are denoted by $\mathbf{q}_{\tau}, \tau=1, \cdots, L$. Note that $\mathbf{p}_{i, l}=\mathbf{q}_{I(i, l)}$, which follows from the definition of index-function (17).

For the NC coefficient vector $\mathbf{g}_{j, l^{\prime}}$, define a support set

$$
\mathcal{S}\left(\mathbf{g}_{j, l^{\prime}}\right) \triangleq\left\{\tau \in\{1, \cdots, L\}: g_{j, l^{\prime}}[\tau] \neq 0\right\}
$$

where $g_{j, l^{\prime}}[\tau]$ denotes the $\tau$ th entry of $\mathbf{g}_{j, l^{\prime}}$. The indices of the non-zero elements are collected in $\mathcal{S}\left(\mathbf{g}_{j, l^{\prime}}\right)$.

We propose to align the signal-spaces of the spatial streams indexed by the non-zero elements of $\mathbf{g}_{j, l^{\prime}}$. Let $\mathbf{a} \| \mathbf{b}$ stands for that $\mathbf{a}$ and $\mathbf{b}$ are parallel in the vector space. The SSA criterion is given as follows.

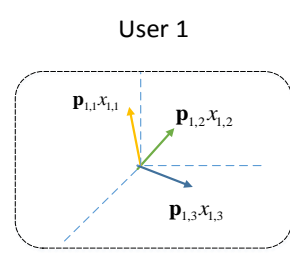

User 2

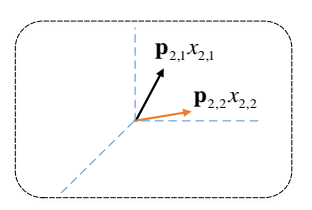

BS 1

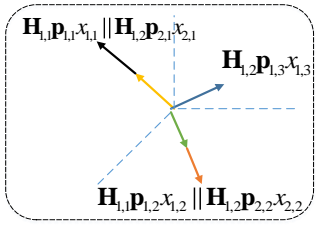

BS 2

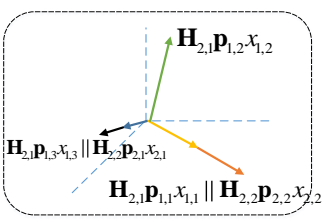

Fig. 3. Illustration of signal-space alignment for a 2-user $2^{\prime}$-BS uplink DMIMO system.

Criterion 1 (SSA precoding): For BS $j$, the signal-spaces of the spatial streams w.r.t. $\mathbf{g}_{j, l^{\prime}}$ are aligned if

$$
\mathbf{H}_{j, I^{-1}(\tau)} \mathbf{q}_{\tau} \| \mathbf{H}_{j, I^{-1}\left(\tau^{\prime}\right)} \mathbf{q}_{\tau^{\prime}} \forall\left(\tau, \tau^{\prime}\right) \in \mathcal{S}\left(\mathbf{g}_{j, l^{\prime}}\right) .
$$

Example 1: Consider $K=K^{\prime}=2, N=N^{\prime}=3$. User 1 and User 2 have $L_{1}=3$ and $L_{2}=2$ spatial streams, respectively. 
There are totally $L=5$ spatial streams. BS 1 and BS 2 want to generate $L_{1}^{\prime}=3$ and $L_{2}^{\prime}=2 \mathrm{NC}$ message sequences, respectively. We arbitrarily consider that the NC generator matrix being

$$
\mathbf{G}=\left[\begin{array}{l}
\mathbf{G}_{1} \\
\mathbf{G}_{2}
\end{array}\right]=\underbrace{\left[\begin{array}{ccccc}
1 & 0 & 0 & 1 & 0 \\
0 & 1 & 0 & 0 & 1 \\
0 & 0 & 1 & 0 & 0 \\
0 & 0 & 1 & 1 & 0 \\
1 & 0 & 0 & 0 & 1
\end{array}\right]}_{L=L_{1}+L_{2}=5}\} L^{\prime}=L_{1}^{\prime}+L_{2}^{\prime}=5 .
$$

The SSA w.r.t. the pattern given by $\mathbf{G}$ is depicted in Fig. 3 . Consider the first NC message to be computed by BS 1 . The NC coefficient vector is $\mathbf{g}_{1,1}=\left[\begin{array}{lllll}1 & 0 & 0 & 1 & 0\end{array}\right]$. The non-zero elements $\mathbf{g}_{1,1}$ have positions

$$
\mathcal{S}\left(\mathbf{g}_{1,1}\right)=\{1,4\} .
$$

Let $\tau=1$ and $\tau^{\prime}=4$. The user indices are respectively $I^{-1}(\tau=1)=1$ and $I^{-1}\left(\tau^{\prime}=4\right)=2$. According to the criterion of the SSA in Eq. (24), the precoding matrices are designed to satisfy

$$
\mathbf{H}_{1,1} \mathbf{q}_{1} \| \mathbf{H}_{1,2} \mathbf{q}_{4}
$$

which is equivalent to

$$
\mathbf{H}_{1,1} \mathbf{p}_{1,1} \| \mathbf{H}_{1,2} \mathbf{p}_{2,1} \text {. }
$$

Now the first spatial stream of User 1 and the first spatial stream of User 2 are aligned at BS 1 .

Likewise, the alignment conditions w.r.t. all other rows of $\mathbf{G}$ can be obtained as follows

$\mathbf{H}_{1,1} \mathbf{p}_{1,2}\left\|\mathbf{H}_{1,2} \mathbf{p}_{2,2}, \quad \mathbf{H}_{2,1} \mathbf{p}_{1,3}\right\| \mathbf{H}_{2,2} \mathbf{p}_{2,1}, \mathbf{H}_{2,1} \mathbf{p}_{1,1} \| \mathbf{H}_{2,2} \mathbf{p}_{2,2}$.

In this way, the SSA precoding matrices $\mathbf{P}_{1}, \mathbf{P}_{2}$ is obtained according to the pattern given by $\mathbf{G}$.

Remark 5: Although there are totally $L=\sum_{i=1}^{K} L_{i}$ spatial streams, by using the SSA precoding, there are only up to $L_{j} \leq N_{j}^{\prime}$ signal directions in the receive signal-space at BS $j$. In particular, some spatial streams are aligned to the same signalspace at the receiver, according to the pattern given by $\mathbf{G}$.

The SSA precoding according to Criterion 1 does not always exist. The existence of SSA precoding is related to the number of antennas, the number of users and the number of base stations. We will discuss this issue later in Section IV. C of this paper.

We next consider a subclass of the SSA precoding, referred to as scaled SSA precoding.

Criterion 2 (Scaled SSA precoding): The scaled SSA precoding satisfies

$$
\begin{aligned}
& \mathbf{H}_{j, I^{-1}(\tau)} \mathbf{q}_{\tau} \| \mathbf{H}_{j, I^{-1}\left(\tau^{\prime}\right)} \mathbf{q}_{\tau^{\prime}} \\
& \frac{\left\|\mathbf{H}_{j, I^{-1}(\tau)} \mathbf{q}_{\tau}\right\|}{\left\|\mathbf{H}_{j, I^{-1}\left(\tau^{\prime}\right)} \mathbf{q}_{\tau^{\prime}}\right\|}=\frac{g_{j, l^{\prime}}[\tau]}{g_{j, l^{\prime}}\left[\tau^{\prime}\right]}, \forall\left(\tau, \tau^{\prime}\right) \in \mathcal{S}\left(\mathbf{g}_{j, l^{\prime}}\right)
\end{aligned}
$$

As we will see later, the quantization error due to the integerpenalty in CF is removed [16] by using the scaled SSA. If the entries of the NC generator matrix $\mathbf{G}$ are belong to $\{0,1\}$, the criterion becomes

$$
\mathbf{H}_{j, I^{-1}(\tau)} \mathbf{q}_{\tau}=\mathbf{H}_{j, I^{-1}\left(\tau^{\prime}\right)} \mathbf{q}_{\tau^{\prime}} \forall\left(\tau, \tau^{\prime}\right) \in \mathcal{S}\left(\mathbf{g}_{j, l^{\prime}}\right) .
$$

The feasibility of the SSA will be presented in Section IV. C.

We note that our proposed approach is different from the integer-forcing linear receiver [33] and integer-forcing IA schemes [22]. The integer-forcing linear receiver is concerned with the MIMO detection, where no efforts are made for managing the signal-spaces for the spatial streams. The integer-forcing interference alignment is developed for the interference channel. It considered decoding integer combinations of the interferences in the waste bin, which is then successively canceled to make the desired signal "cleaner", thus achieving a higher rate in the interference channel with IA. In contrast, our proposed is based on a pre-assigned NC generator matrix for SSA, particularly developed for DMIMO system with rate-constrained $\mathrm{BH}$.

Our proposed signal-space alignment aided network coding scheme differs from the channel integer alignment scheme in [34] in the following aspects: First, at the heart of our proposed scheme lies a network coding generator matrix, which determines the pattern of signal-space alignment. The existence of the network coding generator matrix is a pre-requisite for implementing signal space alignment in the DMIMO system. This is not relevant in [34]. Second, [34] considered two-user cognitive interference network and a two-user Gaussian interference channel, which are different system models. Note that our proposed scheme applies to a general K-user system, while that in [34] only apply to a two-user system.

\section{Base Stations' Processing and Physical-layer Network Coding}

1) Receiver Shaping: BS $j$ carries out a linear transformation which shapes the received signal, given as

$$
\mathbf{T}_{j}=\mathbf{F}_{j} \mathbf{Y}_{j}, j=1, \cdots, K^{\prime} .
$$

Denote by $\mathbf{f}_{j, l^{\prime}}$ and $\mathbf{t}_{j, l^{\prime}}$ the $l^{\prime}$-th row of $\mathbf{F}_{j}$ and $\mathbf{T}_{j}$, respectively. The $l^{\prime}$-th NC message of BS $j$ is computed based on $\mathbf{t}_{j, l^{\prime}}$. We consider the following criterion for the shaping matrix $\mathbf{F}_{j}$ :

Criterion 3 (Zero-forcing receiver shaping): The row vector $\mathbf{f}_{j, l^{\prime}},\left\|\mathbf{f}_{j, l^{\prime}}\right\|=1$, projects the received signal $\mathbf{Y}_{j}$ to the subspace which is perpendicular to the signal-spaces of those signals that are irrelevant to the computation of the $l$ th NC message.

Recall the support set $\mathcal{S}\left(\mathbf{g}_{j, l^{\prime}}\right)$ defined earlier. Let $s_{1}\left(j, l^{\prime}\right)$ be the first element of $\mathcal{S}\left(\mathbf{g}_{j, l^{\prime}}\right)$. Then, the zero-forcing is given by

$$
\mathbf{f}_{j, l^{\prime}}: \mathbf{f}_{j, l^{\prime}} \mathbf{H}_{j, 1} \mathbf{p}_{1, \eta}=0 \forall \eta \in\left\{1, \cdots, L_{1}^{\prime}\right\}, \eta \neq s_{1}\left(j, l^{\prime}\right) .
$$

Example 2: Consider the case in Example 1. Let us first consider $l^{\prime}=1, j=2$, The rows of $\mathbf{F}_{2}$ is obtained as follows. By observing the NC coefficient vector $\mathbf{g}_{2,1}$, we obtain

$$
\mathcal{S}\left(\mathbf{g}_{2,1}\right)=\{3,4\} \text {. }
$$

The first element of $\mathcal{S}\left(\mathbf{g}_{2,1}\right)$ is $s_{1}\left(j, l^{\prime}\right)=3$. From Eq. (33), we have $\eta \in\{1,2\}$, and $\mathbf{f}_{2,1}$ satisfies

$$
\mathbf{f}_{2,1}\left[\mathbf{H}_{2,1} \mathbf{p}_{1,1}, \mathbf{H}_{2,1} \mathbf{p}_{1,2}\right]=0 .
$$

In other words, $\mathbf{f}_{2,1}$ lies in the null space of $\left[\mathbf{H}_{2,1} \mathbf{p}_{1,1}, \mathbf{H}_{2,1} \mathbf{p}_{1,2}\right]$. Likewise, it follows that

$$
\mathbf{f}_{2,2}\left[\mathbf{H}_{2,1} \mathbf{p}_{1,2}, \mathbf{H}_{2,1} \mathbf{p}_{1,3}\right]=0 .
$$


In a similar fashion, the rows of shaping matrix $\mathbf{F}_{1}$ for BS 1 can be obtained as:

$$
\begin{aligned}
& \mathbf{f}_{1,1}\left[\mathbf{H}_{1,1} \mathbf{p}_{1,2}, \mathbf{H}_{1,1} \mathbf{p}_{1,3}\right]=0, \mathbf{f}_{1,2}\left[\mathbf{H}_{1,1} \mathbf{p}_{1,1}, \mathbf{H}_{1,1} \mathbf{p}_{1,3}\right]=0, \\
& \mathbf{f}_{1,3}\left[\mathbf{H}_{1,1} \mathbf{p}_{1,1}, \mathbf{H}_{1,1} \mathbf{p}_{1,2}\right]=0 .
\end{aligned}
$$

2) Physical-layer Network Coding: At BS $j$, after the receiver shaping with zero-forcing, the $L_{j}^{\prime}$ signal sequences $\mathbf{t}_{j, 1}, \cdots, \mathbf{t}_{j, L_{j}^{\prime}}$ are non-interfering where each involves a number of aligned spatial streams. Based on $\mathbf{t}_{j, 1}, \cdots, \mathbf{t}_{j, L_{j}^{\prime}}$, BS $j$ employs $L_{j}^{\prime}$ independent PNC operations to compute the $L_{j}^{\prime}$ NC message sequences, respectively. Recall the $L_{j}^{\prime}$ NC message sequences to be generated at $\mathrm{BS} j$ represented by

$$
\mathbf{u}_{j, l^{\prime}}=\mathbf{g}_{j, l^{\prime}} \otimes \mathbf{B}, l^{\prime}=1, \cdots, L_{j}^{\prime} .
$$

The $L_{j}^{\prime}$ independent PNC operations at BS $j$ can be written as

$$
\mathbf{t}_{j, l^{\prime}} \stackrel{P N C}{\rightarrow} \widehat{\mathbf{u}}_{j, l^{\prime}}, l^{\prime}=1, \cdots, L_{j}^{\prime} .
$$

The computed NC messages sequences are forwarded to the $\mathrm{NC}$ via the $\mathrm{BH}$ of $\mathrm{BS} j$. Note that each spatial stream only appears in one of the $\mathbf{t}_{j, 1}, \cdots, \mathbf{t}_{j, L_{j}^{\prime}}$ sequences at each BS. Therefore, each spatial stream is involved in no more than one NC message sequence at each $\mathrm{BS}$.

The notion of PNC in the seminal paper [35] is to directly obtain a binary sum of the bits of two users, without completely distinguishing each individual user's message bit. The information theoretic result on PNC was reported in [30], where PNC is shown to achieve the capacity of Gaussian two-way relay channel within $1 / 2$ bit, and has a doubled DoF compared to the complete decoding. The CF strategy generalized the above result to scenarios with multiple users, multiple relays, and fading channels. Nested lattice codes were used as a mathematical tool to prove the information theoretic results. From a practical aspect, our group has developed a random-coset non-binary irregular repeat accumulate coded PNC scheme with iterative believe propagation decoding, which approaches the capacity limit within a fraction of $\mathrm{dB}$ [31], [32]. These PNC notions and practical techniques can be employed to accomplish the task in Eq. (36).

\section{Final Decoding at $C U$}

1) Backhaul: Here we first consider that the BSs forward their $\mathrm{NC}$ message sequences to the $\mathrm{CU}$ independently, i.e., without using distributed source coding (such as Slepian-wolf coding) among BSs. The length of the $l^{\prime}$ th NC message sequence at BS $j$ is given by

$$
\max _{i, l: g_{j, l^{\prime}}[I(i, l)] \neq 0} m_{i}^{l}, l^{\prime}=1, \cdots, L_{j}^{\prime}, j=1, \cdots, K^{\prime} .
$$

Here, $i, l: g_{j, l^{\prime}}[I(i, l)] \neq 0$ finds all message streams that are contained in $l^{\prime}$ th NC message sequence at $\mathrm{BS} j$.

The sum of the entropies of the NC message sequences are given by

$$
\sum_{j=1}^{K^{\prime}} H\left(\mathbf{U}_{j}\right)=\sum_{j=1}^{K^{\prime}} \sum_{l^{\prime}=1}^{L_{i}^{\prime}} \max _{i, l: g_{j, l^{\prime}}[I(i, l)] \neq 0} m_{i}^{l} \log _{2} q
$$

where we have used the fact that the rows of $\mathbf{U}_{j}$ are statistically independent in the proposed scheme.
Then, from (4), all NC messages $\widehat{\mathrm{U}}$ are delivered to the $\mathrm{CU}$ error-free if

$$
\sum_{j=1}^{K^{\prime}} \sum_{l^{\prime}=1}^{L_{i}^{\prime}} \max _{i, l: g_{j, l^{\prime}}[I(i, l)] \neq 0} m_{i}^{l} \log _{2} q<C_{B H} .
$$

We next consider distributed source coding among BSs is used in forwarding the NC message sequences. From the Slepian-wolf coding theorem, all NC messages can be delivered to the $\mathrm{CU}$ error-free if [29]

$$
\begin{aligned}
& H\left(\mathbf{U}_{1}, \cdots, \mathbf{U}_{K^{\prime}}\right) \stackrel{(a)}{=} H\left(\mathbf{B}_{1}, \cdots, \mathbf{B}_{K}\right) \\
= & \sum_{i=1}^{K} \sum_{l=1}^{L_{i}} m_{i}^{l} \log _{2} q<C_{B H}
\end{aligned}
$$

where in " $(a)$ " we have used the one-to-one mapping $\mathbf{U}=$ $\mathbf{G} \otimes \mathbf{B}$. We note the parameters $L_{i}$ and $L_{j}^{\prime}$ can be adjusted to the meet the $\mathrm{BH}$ rate constraint.

2) Decoding by Solving Linear Equations: Let the computed $\mathrm{NC}$ message sequences by all BSs be collectively denoted by $\widehat{\mathbf{U}}=\left[\widehat{\mathbf{U}}_{1}^{T}, \cdots, \widehat{\mathbf{U}}_{K^{\prime}}^{T}\right]^{T}$. Upon obtaining $\widehat{\mathbf{U}}$, the CU recovers all users' messages by

$$
\widehat{\mathbf{B}}=\mathbf{G}^{-1} \otimes \widehat{\mathbf{U}}
$$

provided that $\mathbf{G}$ is of full rank. It is obvious that $\widehat{\mathbf{B}}=\mathbf{B}$ if and only if $\widehat{\mathbf{U}}=\mathbf{U}$.

We note that the proposed coding scheme in this paper cannot be applied to the downlink DMIMO system. For the uplink scenario considered in this paper, for each aligned signal-space, a superposition of multiple streams is observed. PNC (or CF) is used to directly reconstruct the linear message combinations, which leads to significant coding gain or even greater DoF than complete decoding all individual streams. The CU can solve those linear message combinations and eventually recover all users' messages. Yet, in the downlink scenario, the new challenge is that the user nodes, where PNC or CF is conducted, are already the final destinations. Thus, the computed linear message combinations at each user node have to be identical to the desired messages. New coding schemes need to be studied to address this challenge for the downlink scenario. This is out of the scope of this paper and is studied in [36].

\section{Achievable Rates of the Proposed Scheme}

In this section, we analyze the proposed scheme. We assume that $N_{1}=N_{2}=\cdots=N_{K}=N$ and $N_{1}^{\prime}=N_{2}^{\prime}$ $=\cdots=N_{K^{\prime}}^{\prime}=N^{\prime}$ throughout this section. Our treatment can be straightforwardly extended to the case where the wireless terminals have different numbers of antennas.

In this paper, we consider that all the entries of the precoding matrices obtained above are divided by a factor of $\sum_{i=1}^{K} \operatorname{Tr}\left(\mathbf{P}_{i} \mathbf{P}_{i}^{H}\right) / E_{\text {total }}$. The resultant precoding matrices always satisfy the power constraint (3), which will not be mentioned in the sequel to avoid redundancy. 


\section{A. Properties and Existence of NC Generator Matrix $\mathbf{G}$}

In the previous section, we have seen that the $\mathrm{NC}$ generator matrix $\mathbf{G}$ determines the pattern of the SSA, and thus the precoding matrices $\mathbf{P}_{i}, i=1, \cdots, K$. On the other hand, the implementation of the SSA also imposes some structural properties on G, summarized below. Consider a $\left(K, K^{\prime}, N, N^{\prime}\right)$ DMIMO system where the total number of operating spatial streams is $L$, $L \leq \min \left(K^{\prime} N^{\prime}, K N\right){ }^{3}$

Property 1: The number of rows of $\mathbf{G}_{j}$, i.e. $L_{j}^{\prime}, j \in$ $\left\{1, \cdots, K^{\prime}\right\}$, is no greater than $N^{\prime}$.

Explanation: For each BS, the total receive signal-space has a dimension of $N^{\prime}$. As such, no more than $N^{\prime}$ aligned signalspaces can be created by the proposed. Thus, the number of NC message sequences to be computed at each BS, denoted by $L_{j}^{\prime}$, is no greater than $N^{\prime}$.

Define the Hamming weight of a vector as the total number non-zero entries.

Property 2: a) For BS $j$ such that $L_{j}^{\prime}=N^{\prime}$, the Hamming weight of a column of $\mathbf{G}_{j}, j \in\left\{1, \cdots, K^{\prime}\right\}$, is 1 . (That is, each column of $\mathbf{G}_{j}$ contains one non-zero entry.) Also, the sum of the Hamming weights of the rows of $\mathbf{G}_{j}$ is equal to $L$.

b) For BS $j$ such that $L_{j}^{\prime}<N^{\prime}$, the Hamming weight of a column of $\mathbf{G}_{j}, j \in\left\{1, \cdots, K^{\prime}\right\}$, is 1 or 0 . The sum of the Hamming weights of the rows of $\mathbf{G}_{j}, j \in\left\{1, \cdots, K^{\prime}\right\}$, is no greater than $L$.

Explanation: a) Recall that in the proposed scheme, if $L_{j}^{\prime}=$ $N^{\prime}$, each spatial stream is involved exactly one NC message sequence at BS $j$. Therefore, each column of $\mathbf{G}_{j}$ contains one non-zero entry. In addition, the sum of the Hamming weights of the rows of $\mathbf{G}_{j}$ is equal to $L$, as there are $L$ spatial streams in total.

Property 3: The rank of $\mathbf{G}$ is $L$ in $\mathrm{GF}(q)$.

We next present a proposition on the existence of a square matrix $\mathbf{G}$ satisfying all the above properties.

Proposition 1: There exists a NC generator matrix $\mathbf{G}$ satisfying Properties 1-3 as long as the entries of $\mathbf{G}$ belongs to $\{0,1, \ldots, p-1\}, p=3$, for any $L \leq K^{\prime} N^{\prime}$. (See proof in Appendix.)

Remark 6: Proposition 1 suggests that we can construct the matrix $\mathbf{G}$ with entries only in $\{0,1,2\}$.

\section{B. An Achievable Rate Conditioned on Aligned Signal-spaces}

Based on the existence of the NC generator matrix, we next present our result on the achievable rate of the proposed scheme, given that the signal-space has been aligned according to the pattern given by $\mathbf{G}$.

Let

$$
\eta_{i, l}=l^{\prime}: g_{j, l^{\prime}}[I(i, l)] \neq 0
$$

\footnotetext{
${ }^{3}$ Note that our scheme applied to the case where the terminals have different numbers of antennas as illustrated in Sections II and III. Here we consider the case with equal number of antennas primarily for a more concise way of presenting the achievable rate result of the propose scheme.
}

Proposition 2: The following rate-tuple

$$
\begin{aligned}
& R_{i}=\sum_{l=1}^{L_{i}} R_{i, l}<\sum_{l=1}^{L_{i}} \min _{j \in\left\{1, \cdots, K^{\prime}\right\}} \log _{2}\left(\left|\mathbf{f}_{j, \eta_{i, l}} \mathbf{H}_{j, i} \mathbf{p}_{i, l}\right|^{2} \rho\right)^{+} \log _{2} q \\
& i \in\{1, \cdots, K\} \\
& \sum_{i=1}^{K} R_{i}<\bar{C}_{B H}
\end{aligned}
$$

is achievable, if $\mathbf{p}_{i, l}, l=1, \cdots, L_{i}, i=1, \cdots, K$, satisfy the SSA condition specified in Eq. (30).

Proof. Without loss of generality, let the $L$ coded-modulated streams $\mathbf{c}_{i, l}, l=1, \ldots, L_{i}, i=1, \ldots, K$,be arranged in a descending order according their rates. Consider a chain of lattices $\Lambda \subseteq \Lambda_{L} \subseteq \cdots \subseteq \Lambda_{1}$. The coarse lattice $\Lambda$ is employed as the shaping lattice which is common to all $L$ data streams. The $L$ fine lattices $\Lambda_{1}, \ldots, \Lambda_{L}$ are used for channel coding for the $L$ data streams, respectively. The coarse lattice is choosen to be simultaneously Rogers-good and Poltyrev-good, while the fine lattices are Poltyrev-good [30].

Consider that the entries of $\mathbf{G}$ belong to $\{0,1,2\}$. From Proposition 1, there exists a $\mathbf{G}$ that satisfies Properties 1-3 for any $L<N^{\prime} K^{\prime}$. Consider the $\eta_{i, l}=l^{\prime}$ such that the $I(i, l)$-th element of $\mathbf{g}_{j}^{l^{\prime}}$ is non-zero. That is, the $l^{\prime}$ th NC message sequence of BS $j$ contains the $l$ th message stream of User $i$. The signal-space of the $l$ th spatial stream of User $i$ is $\mathbf{H}_{j, i} \mathbf{p}_{i, l}$ or $\mathbf{H}_{j, i} \mathbf{q}_{\tau}, \tau=I(i, l)$. Consider the scaled SSA condition is met, there are some other spatial streams whose signal-spaces are identical to $\mathbf{H}_{j, i} \mathbf{q}_{\tau}$, i.e.,

$g_{j, l^{\prime}}\left[\tau^{\prime}\right] \mathbf{f}_{j, l^{\prime}} \mathbf{H}_{j, i} \mathbf{q}_{\tau}=g_{j, l^{\prime}}[\tau] \mathbf{f}_{j, l^{\prime}} \mathbf{H}_{j, I^{-1}\left(\tau^{\prime}\right)} \mathbf{q}_{\tau^{\prime}}, \forall \tau^{\prime} \neq \tau, \tau^{\prime} \in \mathcal{S}\left(\mathbf{g}_{j, l^{\prime}}\right)$

after the zero-forcing receiver shaping. BS $j$ computes the following NC message sequence

$$
\mathbf{u}_{j, l^{\prime}}=\mathbf{g}_{j, l^{\prime}} \otimes \mathbf{B}, l^{\prime}=1, \ldots, L_{j}^{\prime},
$$

as in [30], [16]. It has been shown that there exists a nested lattice chain of rate $R_{i, l}^{(j)}$ for encoding $\mathbf{b}_{i, l}$ into $\mathbf{c}_{i, l}$, whose error probability of computing $\mathbf{u}_{j, l^{\prime}}$ vanishes as $n \rightarrow \infty$ as long as [30], [16]

$$
R_{i, l}^{(j)}<\log _{2}\left(\left|\mathbf{f}_{j, l^{\prime}} \mathbf{H}_{j, i} \mathbf{p}_{i, l}\right|^{2} \rho\right)^{+} \log _{2} q, \forall(l, i): g_{j, l^{\prime}}[I(l, i)] \neq 0
$$

Note that owing to the scaled SSA, the quantization error terms as in [16] due to non-integer penalty is removed, and the effective SNR is given by $\left|\mathbf{f}_{j, l^{\prime}} \mathbf{H}_{j, i} \mathbf{p}_{i, l}\right|^{2} \rho$. Taking the minimum of the rates $R_{i, l}^{(1)}, \ldots, R_{i, l}^{\left(K^{\prime}\right)}$ over all BSs, all NC message sequences that contain the $l$ th stream of User $i$ can be reliably computed if

$$
R_{i, l}<\min _{j \in\left\{1, \cdots, K^{\prime}\right\}} R_{i, l}^{(j)}=\min _{j \in\left\{1, \cdots, K^{\prime}\right\}} \log _{2}\left(\left|\mathbf{f}_{j, l^{\prime}} \mathbf{H}_{j, i} \mathbf{p}_{i, l}\right|^{2} \rho\right)^{+} \log _{2} q .
$$

Consider all spatial streams of User $i$, the related NC messages sequences can be reliably computed if the rate in (43) is met. Finally, recall that the BH consumption is given by (40) using Slepian-Wolf coding, which is equivalent to (44). Thus, the rate in (43) is achievable if the BH capacity meets (44). 


\section{On the Feasibility of SSA}

Let $\widetilde{\mathbf{H}}$ be a matrix composed in a block-wise fashion as follows:

Denote the elements of $\mathcal{S}\left(\mathbf{g}_{j, l^{\prime}}\right)$ by $\tau_{1}, \tau_{2}, \ldots, \tau\left|\mathcal{S}\left(\mathbf{g}_{j, l^{\prime}}\right)\right| \cdot$. Consider the first $\mathrm{NC}$ message sequence to be computed at BS 1, i.e., $l^{\prime}=1, j=1$. Then, the first $N^{\prime}$-rows are given by

$$
\left[\mathbf{0}, \ldots, g_{1,1}\left(\tau_{2}\right) \mathbf{H}_{1, I^{-1}\left(\tau_{1}\right)}, \mathbf{0}, \ldots, g_{1,1}\left(\tau_{1}\right) \mathbf{H}_{1, I^{-1}\left(\tau_{2}\right)}, \mathbf{0}, \ldots, \mathbf{0}\right] \text {. }
$$

The second $N^{\prime}$-rows are given by

$$
\left[\mathbf{0}, \ldots, g_{1,1}\left(\tau_{3}\right) \mathbf{H}_{1, I^{-1}\left(\tau_{2}\right)}, \mathbf{0}, \ldots, g_{1,1}\left(\tau_{2}\right) \mathbf{H}_{1, I^{-1}\left(\tau_{3}\right)}, \mathbf{0}, \ldots, \mathbf{0}\right] .
$$

Likewise, the following several $N^{\prime}$-rows of $\widetilde{\mathbf{H}}$ can be obtained. Repeating the above process for all $j=1, \cdots, K^{\prime}, l^{\prime}=$ $1, \cdots, L^{\prime}$, the matrix $\mathbf{H}$ is obtained.

With $\widetilde{\mathbf{H}}$, the criterion of the scaled SSA becomes

$$
\widetilde{\mathbf{H}} \boldsymbol{\theta}=\mathbf{0}
$$

where

$$
\boldsymbol{\theta}=\left[\mathbf{p}_{1,1}^{T}, \cdots, \mathbf{p}_{1, L_{1}}^{T}, \mathbf{p}_{2,1}^{T}, \cdots, \mathbf{p}_{K, L_{K}}^{T}\right]^{T} .
$$

Then, the SSA is feasible if the null space of $\widetilde{\mathbf{H}}$ is not empty.

We obtain the following result on the feasibility of SSA.

Proposition 3: For a given NC generator matrix G satisfying Property 1-3, the corresponding signals-space alignment criterion can be guranteed if $L$ satisfies

$$
N>\frac{L-N^{\prime}}{L} \bar{K}^{\prime} N^{\prime}, \bar{K}^{\prime}=\left\lceil\frac{L}{N^{\prime}}\right\rceil .
$$

Proof. First consider that $\bar{K}^{\prime}=K^{\prime}$. It can be shown that the dimension of $\widetilde{\mathbf{H}}$ is $\left(L-N^{\prime}\right) N^{\prime} K^{\prime}$ by $L N$. The null space of $\widetilde{\mathbf{H}}$ is not empty if

$$
L N>\left(L-N^{\prime}\right) K^{\prime} N^{\prime}
$$

which yields Eq. (52), no matter $\widetilde{\mathbf{H}}$ is full rank or not. Then, there exists at least one solution which satisfies the SSA criterion in (30). Consider next $\bar{K}^{\prime}=\left\lceil\frac{L}{N^{\prime}}\right\rceil<K^{\prime}$. In such cases, $\bar{K}^{\prime}$ active BSs are sufficient for generating $L \mathrm{NC}$ message sequences. The condition to ensure that $\widetilde{\mathbf{H}}$ matrix is not over-determined for a $\bar{K}^{\prime}$-BS system is simply $L N>\left(L-N^{\prime}\right) \bar{K}^{\prime} N^{\prime}$. This completes the proof of the proposition.

Prop. 3 reveals the relationship between the existence of SSA precoding according to Criterion 1 and the numbers of antennas, the number of users and the number of base stations. Note that the SSA precoding exists according to Prop. 3 even if there is antenna correlation. This is because antenna correlation will only increase the probability of the matrix $\widetilde{\mathbf{H}}$ being rank deficient, which will not affect the existence of solution to Eq. (50).

\section{An Achievable Rate of the Proposed Scheme}

We are now ready to present the following result on the achievable rate based on Propositions 1-3.
Theorem 1: An achieveable rate-tuple is given by

$$
R_{i}<\sum_{l=1}^{L_{i}} R_{i, l}=\sum_{l=1}^{L_{i}} \min _{j \in\left\{1, \cdots, K^{\prime}\right\}} \log _{2}\left(\left|\mathbf{f}_{j, \eta_{i, l}} \mathbf{H}_{j, i} \mathbf{p}_{i, l}\right|^{2} \rho\right)^{+} \log _{2} q,
$$

$i \in\{1, \cdots, K\}$,

$\sum_{i=1}^{K} R_{i}<\bar{C}_{B H}$

with $\mathbf{p}_{i, l}$ satisfying the condition specified in Eq. (30), if the total number of spatial streams $L$ satisfies

$$
L<\frac{\left(N^{\prime}\right)^{2} \bar{K}^{\prime}}{\bar{K}^{\prime} N^{\prime}-N}, \bar{K}^{\prime}=\left\lceil\frac{L}{N^{\prime}}\right\rceil .
$$

\section{E. Relation to Interference Alignment}

In the DMIMO scenario, the interference alignment scheme is a subclass of our proposed scheme. Specifically, our proposed scheme exactly becomes the interference alignment scheme if we choose the $\mathrm{NC}$ generator matrix as follows. Consider $K^{\prime}=$ $K$. User $i$ transmits $L_{i}$ spatial streams, and BS $j, j=i$, is set to decode User $i$ 's spatial streams. Note that $L_{i}^{\prime}=L_{i}$. The corresponding $\mathrm{NC}$ generator matrix w.r.t. the IA scheme is

$$
\begin{gathered}
\left.\mathbf{G}_{i}^{I A}=\left[\begin{array}{cccccccc}
0 & \cdots & 1 & 0 & \cdots & 0 & 0 & \cdots \\
0 & 0 & \cdots & 1 & 0 & \cdots & 0 & \cdots \\
0 & 0 & 0 & \cdots & \ddots & \cdots & 0 & \vdots \\
0 & 0 & 0 & \cdots & 0 & 1 & 0 & \cdots
\end{array}\right]\right\} L_{i} \text { rows, } i=1, \ldots, K \\
\mathbf{G}^{I A}=\left[\left(\mathbf{G}_{1}^{I A}\right)^{T}, \cdots,\left(\mathbf{G}_{K}^{I A}\right)^{T}\right]
\end{gathered}
$$

where the $I(i, l)$ th entry of the $l$ th row of $\mathbf{G}_{i}^{I A}$ is 1 while other entries of that row are zero. It is obvious that $\mathrm{G}^{I A}$ satisfies the structural Properties 1-3.

Therefore, the DoF of our proposed scheme is no smaller than that of the IA scheme in the DMIMO setting. By choosing a better $\mathbf{G}$, improved performance over that with IA can be achieved, as we will see in the next section. This holds even when symbolextension over time-varying channels is taken into consideration.

We note that the proposed method and achievable rate result in this paper does not directly apply to the downlink scenario. First, the design of the network coding generator matrix $G$ will be different. In particular, the network coding generator matrix for the downlink scenario must be designed such that its inverse can guarantee that the spatial streams, which contains the network coded version of the messages, can be desirably aligned at the User nodes. Second, in the downlink case, the achievable rate analysis differs from that of the uplink case, since the relationship between the message rate and the computation rate will be different.

\section{Parallel AND Tri Relay Networks}

In this section, we study the DMIMO systems with $K^{\prime}=2$ and 3 BSs. These setups are also referred to as a parallel relay network and a tri-relay network, respectively. 


\section{A. DoF of DMIMO with Parallel Relay}

We first present a result on the DoF of the scheme.

Theorem 2: Consider $C_{B H}=L \log \rho+\epsilon$, where $\epsilon=o(1)$. For $L \leq \min \left(K N, K^{\prime} N^{\prime}\right)-1$, the total DoF of the DMIMO with $K^{\prime}=2 \mathrm{BSs}$ is given by

$$
d_{\text {total }}=L .
$$

Proof. The converse follows directly from that max-flow min-cut theorem for a general network. Due to the rate-constrained $\mathrm{BH}$, the maximum information rate is no more than $L \log \rho+o(1)$.

We next consider the achievability. Using Proposition 3, it can be shown that

$$
N>\frac{L-N}{L} \bar{K}^{\prime} N^{\prime}
$$

is met for any $L \leq \min \left(K N, 2 N^{\prime}\right)-1$. Thus, the SSA criterion in (30) is guaranteed. In addition, from Proposition 2, a NC generator matrix G satisfying Properties 1-3 exists for $q=2$. Thus, the rate in Proposition 1 is achievable. The corresponding sum-rate is given by

$$
\sum_{i=1}^{K} \sum_{l=1}^{L_{i}} \min _{j \in\left\{1, \cdots, K^{\prime}\right\}} \log _{2}\left(\left|\mathbf{f}_{j, l^{\prime}} \mathbf{H}_{j, i} \mathbf{p}_{i, l}\right|^{2} \rho\right)^{+} \log _{2} q .
$$

Note that $\mathbf{f}_{j, l^{\prime}} \mathbf{H}_{j, i}$ has non-vanishing values with prolixity 1 . As $\rho \rightarrow \infty$, the DoF (the pre-log factor) is given by $\sum_{i=1}^{K} L_{i}=L$. This completes the proof.

Corollary 1: As $N \rightarrow \infty$, the proposed scheme can asymptotically achieve a $\operatorname{DoF}$ of $\min \left(K N, 2 N^{\prime}\right)$ if $C_{B H} \propto$ $\min (K N, 2 N) \log \rho$.

This result show that, as long as the $\mathrm{BH}$ capacity $C_{B H}$ scales like $\min \left(K N, 2 N^{\prime}\right)$ the DMIMO with parallel relays asymptotically achieves a DoF of $\min \left(K N, 2 N^{\prime}\right)$. This is equivalent to the DoF upper bound given by a $K$-user MIMO multiple-access channel with $2 N^{\prime}$ co-located receiver antennas.

1) Case Study I: In this part, we consider a symmetric setup where $K=K^{\prime}=2, N=N^{\prime}$. We explicitly show an example where a DoF of $L=2 N^{\prime}-1$ is achieved with the proposed scheme with $N^{\prime}=3$.

Consider that User 1 transmits $L_{1}=3$ spatial streams while User 2 transmits $L_{2}=2$ spatial streams. Consider that the NC generator matrix being

$$
\mathbf{G}=\left[\begin{array}{c}
\mathbf{G}_{1} \\
-- \\
\mathbf{G}_{2}
\end{array}\right]=\left[\begin{array}{ccccc}
1 & 0 & 0 & 1 & 0 \\
0 & 1 & 0 & 0 & 1 \\
0 & 0 & 1 & 0 & 0 \\
-- & -- & -- & -- & -- \\
0 & 0 & 1 & 1 & 0 \\
1 & 0 & 0 & 0 & 1
\end{array}\right]
$$

which has full rank of 5 in $\mathrm{GF}(2)$. From (48), the corresponding $\widetilde{\mathbf{H}}$ matrix for implementing SSA is given by

$$
\widetilde{\mathbf{H}}=\left[\begin{array}{ccccc}
\mathbf{H}_{1,1} & \mathbf{0} & \mathbf{0} & \mathbf{H}_{1,2} & \mathbf{0} \\
0 & \mathbf{H}_{1,1} & \mathbf{0} & \mathbf{0} & \mathbf{H}_{1,1} \\
-- & -- & -- & -- & -- \\
\mathbf{0} & \mathbf{0} & \mathbf{H}_{2,1} & \mathbf{H}_{2,2} & \mathbf{0} \\
\mathbf{H}_{2,1} & \mathbf{0} & \mathbf{0} & \mathbf{0} & \mathbf{H}_{2,2}
\end{array}\right]
$$

Note that the third row of $\mathbf{G}$ does not impose a constraint for the alignment, thus it is not related to $\widetilde{\mathbf{H}}$.

The conditions for the signal alignment w.r.t. the given $\mathbf{G}$ matrix is

$$
\widetilde{\mathbf{H}}\left[\mathbf{p}_{1,1}^{T}, \mathbf{p}_{1,2}^{T}, \mathbf{p}_{1,3}^{T}, \mathbf{p}_{2,1}^{T}, \mathbf{p}_{2,2}^{T}\right]^{T}=\mathbf{0}
$$

Since Proposition 3 is met for this case, a solution on the precoding matrices does exist which ensures the SSA with the pattern given by $\mathbf{G}$.

With the precoding matrices obtained from (62), at BS 1, we obtain

$$
\mathbf{H}_{1,1} \mathbf{p}_{1,1}=-\mathbf{H}_{1,2} \mathbf{p}_{2,1} \text { and } \mathbf{H}_{1,1} \mathbf{p}_{1,2}=-\mathbf{H}_{1,2} \mathbf{p}_{2,2}
$$

That is, at BS 1, the signal-space of the first spatial stream of User 1 and that of the first spatial stream of User 2 are aligned, while the signal-space of the second spatial stream of User 1 and that of the second spatial stream of User 2 are aligned. The signal space of the third spatial stream of User 1 is given by $\mathbf{H}_{1,1} \mathbf{p}_{1,3}$.

Next, using the receiver shaping (32), the received signal $\mathbf{Y}_{1}$ is projected to the subspace that is perpendicular to the subspace $\operatorname{span}\left\{\mathbf{H}_{1,1} \mathbf{p}_{1,2}, \mathbf{H}_{1,1} \mathbf{p}_{1,3}\right\}$. Based on that, the NC message sequence

$$
\mathbf{u}_{1,1}=\mathbf{g}_{1,1} \otimes \mathbf{B}=\mathbf{b}_{1,1} \oplus \mathbf{b}_{2,1}
$$

is computed using PNC. This PNC computation process is free of interference from other signals streams in $\operatorname{span}\left\{\mathbf{H}_{1,1} \mathbf{p}_{1,2}, \mathbf{H}_{1,1} \mathbf{p}_{1,3}\right\}$. Similarly, the received signal $\mathbf{Y}_{1}$ is projected to the subspace that is perpendicular to the subspace $\operatorname{span}\left\{\mathbf{H}_{1,1} \mathbf{p}_{1,1}, \mathbf{H}_{1,1} \mathbf{p}_{1,3}\right\}$, and the NC message sequence

$$
\mathbf{u}_{1,2}=\mathbf{g}_{1,2} \otimes \mathbf{B}=\mathbf{b}_{1,2} \oplus \mathbf{b}_{2,2}
$$

is computed using PNC. The received signal $\mathbf{Y}_{1}$ is projected to the subspace that is perpendicular to the subspace $\operatorname{span}\left\{\mathbf{H}_{1,1} \mathbf{p}_{1,1}, \mathbf{H}_{1,1} \mathbf{p}_{1,2}\right\}$, and the message

$$
\mathbf{u}_{1,3}=\mathbf{g}_{1,3} \otimes \mathbf{B}=\mathbf{b}_{1,3}
$$

is decoded. Likewise, at BS 2, the NC message sequences

$\mathbf{u}_{2,1}=\mathbf{g}_{2,1} \otimes \mathbf{B}=\mathbf{b}_{1,3} \oplus \mathbf{b}_{2,1}$ and $\mathbf{u}_{2,2}=\mathbf{g}_{2,2} \otimes \mathbf{B}=\mathbf{b}_{1,1} \oplus \mathbf{b}_{2,2}$

are computed. So far, the $L=5 \mathrm{NC}$ message sequences are computed. These NC message sequences are forwarded to the $\mathrm{CU}$ via the $\mathrm{BH}$ links. If $C_{B H}=5 \log \rho+\epsilon$, these $\mathrm{NC}$ messages can be reliably delivered to the $\mathrm{CU}$, for a sufficiently large $\epsilon=o(1)$. Then the CU can recover all 5 spatial streams by solving the 5 linear equations. The sum DoF is 5 .

For comparison purpose, let us revisit two benchmark schemes. The first one is the orthogonalized transmission, where the two users take turn to transmit. The second one is the a scheme which directly applies the strategy for a interference channel, where BS 1 only decodes the first user's spatial streams while BS 2 only decodes the second user's spatial streams.

For the DMIMO setting with $K^{\prime}=2 \mathrm{BSs}$, the DoFs of both benchmark schemes are equal to $N^{\prime}$ if the BH capacity scales like $C_{B H} \propto N^{\prime} \log \rho$. Yet, as $C_{B H}$ scales at a higher speed, i.e., $C_{B H} \propto L \log \rho, L>N^{\prime}$, none of them can yield increased DoF. In contrast, our proposed scheme yields a DoF of $L$ for $N^{\prime}<L \leq 2 N^{\prime}-1$. Therefore, our propose scheme achieves up to $\frac{2 N^{\prime}-1}{N^{\prime}}$ times of DoF of the benchmark schemes. 
2) Case Study II: In this part, we consider a setup where $K=$ $K^{\prime}=2, N^{\prime}=3$. Consider that User 1 has 3 data streams while User 2 has 3 data streams. There are totally $L=6$ spatial streams. We consider that the network coding generator matrix being

$$
\mathbf{G}=\left[\begin{array}{c}
\mathbf{G}_{1} \\
-- \\
\mathbf{G}_{2}
\end{array}\right]=\left[\begin{array}{cccccc}
2 & 0 & 0 & 1 & 0 & 0 \\
0 & 1 & 0 & 0 & 1 & 0 \\
0 & 0 & 1 & 0 & 0 & 1 \\
-- & -- & -- & -- & -- & -- \\
1 & 0 & 0 & 0 & 1 & 0 \\
0 & 1 & 0 & 0 & 0 & 1 \\
0 & 0 & 1 & 1 & 0 & 0
\end{array}\right]
$$

which has a full rank of 6 in $\operatorname{GF}(q)$. Note that we have extended the Galois Field to $q=3$, as there does not exist a full rank $\mathbf{G}$ satisfying Properties $1-3$ for $q=2$. The corresponding $\widetilde{\mathbf{H}}$ matrix for SSA is given by

$$
\widetilde{\mathbf{H}}=\left[\begin{array}{cccccc}
\mathbf{H}_{1,1} & \mathbf{0} & \mathbf{0} & 2 \mathbf{H}_{1,2} & \mathbf{0} & \mathbf{0} \\
\mathbf{0} & \mathbf{H}_{1,1} & \mathbf{0} & \mathbf{0} & \mathbf{H}_{1,1} & \mathbf{0} \\
\mathbf{0} & \mathbf{0} & \mathbf{H}_{1,1} & \mathbf{0} & \mathbf{0} & \mathbf{H}_{1,1} \\
-- & -- & -- & -- & -- & -- \\
\mathbf{H}_{2,1} & \mathbf{0} & \mathbf{0} & \mathbf{0} & \mathbf{H}_{2,2} & \mathbf{0} \\
\mathbf{0} & \mathbf{H}_{2,1} & \mathbf{0} & \mathbf{0} & \mathbf{0} & \mathbf{H}_{2,2} \\
\mathbf{0} & \mathbf{0} & \mathbf{H}_{2,1} & \mathbf{H}_{2,2} & \mathbf{0} & \mathbf{0}
\end{array}\right] .
$$

The conditions for the SSA w.r.t. the given $\mathbf{G}$ matrix is $\widetilde{\mathbf{H}}\left[\mathbf{p}_{1,1}^{T}, \mathbf{p}_{1,2}^{T}, \mathbf{p}_{1,3}^{T}, \mathbf{p}_{2,1}^{T}, \mathbf{p}_{2,2}^{T}, \mathbf{p}_{2,3}^{T}\right]^{T}=\mathbf{0}$. For $N^{\prime}=3$, from Proposition 3, Eq. (52), a solution does exist as long as one of the users has a number of antennas greater than 3 while the other user has 3 antennas. Then, a DoF of 6 can be achieved as long as $C_{B H} \propto L \log \rho$.

\section{B. On the DoF of DMIMO with Tri-Relay}

In this part, we consider DMIMO systems with three $K^{\prime}=3$ BSs. Let us first discuss the two benchmark schemes where $N=N^{\prime}=3$. For the orthogonal transmission, the DoF is just 3. For the interference channel strategy, it is well-known that interference alignment (IA) can yield greater DoF than the orthogonal transmission. For a block-fading (or constant) channel without symbol extension, a DoF of 4 can be achieved using IA. Specifically, User 1 transmits two spatial streams and each of the other two users transmit one spatial stream. The IA precoders are designed such that at BS 1, the signals from the Users 2 and 3 are aligned in a one-dimension subspace; at BS 2, one spatial stream of User 1 and that of User 3 is aligned;at BS 3, one spatial stream of User 1 and that of User 2 is aligned. In such a manner, a total DoF of 4 can be achieved.

The aforementioned IA strategy can be directly applied in the DMIMO system. However, if the $\mathrm{BH}$ scales like $C_{B H} \propto$ $L \log \rho, L>4$, the DoF using the IA strategy is still 4 .

1) Case Study I: We next show a simple example where our proposed scheme can achieve a DoF of $d_{\text {total }}=5$. Consider that Users 1 and 2 have 2 spatial streams each, and User 3 has 1 spatial stream. There are totally $L=5$ spatial streams. The precoding vectors for these streams are $\mathbf{p}_{1,1}, \mathbf{p}_{1,2}, \mathbf{p}_{2,1}, \mathbf{p}_{2,1}, \mathbf{p}_{3,1}$. Consider the following full rank NC generator matrix

$$
\mathbf{G}=\left[\begin{array}{c}
\mathbf{G}_{1} \\
-- \\
\mathbf{G}_{2} \\
-- \\
\mathbf{G}_{3}
\end{array}\right]=\left[\begin{array}{ccccc}
1 & 0 & 1 & 0 & 0 \\
0 & 1 & 0 & 1 & 0 \\
-- & -- & -- & -- & -- \\
1 & 0 & 0 & 1 & 0 \\
0 & 1 & 0 & 0 & 1 \\
-- & -- & -- & -- & -- \\
0 & 0 & 1 & 0 & 0
\end{array}\right]
$$

The corresponding $\widetilde{\mathbf{H}}$ matrix for implementing the SSA is given by

$$
\widetilde{\mathbf{H}}=\left[\begin{array}{ccccc}
\mathbf{H}_{1,1} & 0 & \mathbf{H}_{1,2} & 0 & 0 \\
0 & \mathbf{H}_{1,1} & 0 & \mathbf{H}_{1,2} & 0 \\
-- & -- & -- & -- & -- \\
\mathbf{H}_{2,1} & 0 & 0 & \mathbf{H}_{2,2} & 0 \\
0 & \mathbf{H}_{2,1} & 0 & 0 & \mathbf{H}_{2,3} \\
-- & -- & -- & -- & -- \\
0 & 0 & \mathbf{H}_{3,2} & 0 & 0
\end{array}\right] .
$$

It can be shown that a solution to $\widetilde{\mathbf{H}}\left[\mathbf{p}_{1,1}^{T}, \mathbf{p}_{1,2}^{T}, \mathbf{p}_{2,1}^{T}, \mathbf{p}_{2,1}^{T}, \mathbf{p}_{3,1}^{T}\right]=0$ exists. Using the similar procedure described in the previous subsection. The $5 \mathrm{NC}$ message sequences w.r.t. $\mathbf{G}$ can be computed, with which the $\mathrm{CU}$ can recover all 5 spatial streams. The DoF is 5 as long as $C_{B H} \propto 5 \log \rho+\epsilon$ for a sufficiently large $\epsilon=o(1)$.

The above example showed that the proposed scheme yields a higher DoF compared to IA. We note that even with symbol extension and time-varying channel, the DoF with IA is at most 4.5 , and our proposed scheme (without symbol extension) still outperform it.

2) Case Study II: Here we study a case where a DoF of $d_{\text {total }}=L=7$ is achieved. Let User 1 transmit 3 spatial streams and each of Users 2 and 3 transmits 2 streams. Consider the following NC generator matrix in (68) which has a full rank of 7. The corresponding $\widetilde{\mathbf{H}}$ matrix for SSA can be obtained as in previous examples.

A solution for the SSA exists if the condition in Proposition 4 is met, which is $7 N>12 N^{\prime}$. That is, more transmit antenna may be required to realize the DoF of 7 in this example. We note that the DoF with IA is at most 4.5 (with infinite symbol-extension) even the above condition on the antenna configuration is met, which is still much smaller than that achieved by our proposed scheme.

$$
\mathbf{G}=\left[\begin{array}{lllllll}
1 & 0 & 0 & 1 & 0 & 1 & 0 \\
0 & 1 & 0 & 0 & 1 & 0 & 0 \\
0 & 0 & 1 & 0 & 0 & 0 & 1 \\
0 & 1 & 0 & 0 & 1 & 0 & 1 \\
1 & 0 & 0 & 1 & 0 & 0 & 0 \\
1 & 0 & 0 & 0 & 1 & 0 & 0 \\
0 & 1 & 0 & 0 & 0 & 1 & 0
\end{array}\right]
$$

\section{Numerical Results}

Here we present numerical results on the achievable sum-rates of the proposed scheme for DMIMO systems with various parameters. In the simulations, we consider Rayleigh fading channel coefficients. The results are obtained by averaging 100,000 fading realizations. Fig. 4 shows the averaged achievable sum-rates of the proposed scheme for a DMIMO system of $K=K^{\prime}=2$, $N^{\prime}=N=3$. Here, the $\mathrm{BH}$ capacity is given by ${ }^{4} C_{B H}$ $=L \log \rho+\epsilon, L=2,3,4,5$. The achievable rate of the benchmark scheme using orthogonal transmission (or the interference channel strategy) where $L \geq 3$ is also plotted. It is demonstrated that the proposed scheme can achieve a much greater sum-rate over the benchmark scheme, due to the higher DoF. It is noteworthy that if one of the users is equipped with 4 transmit antennas, a total DoF of 6 can be achieved, where a even greater gain over the benchmark can be achieved.

\footnotetext{
${ }^{4} \mathrm{~A}$ sufficiently large $\epsilon=o(1)$ is considered.
} 


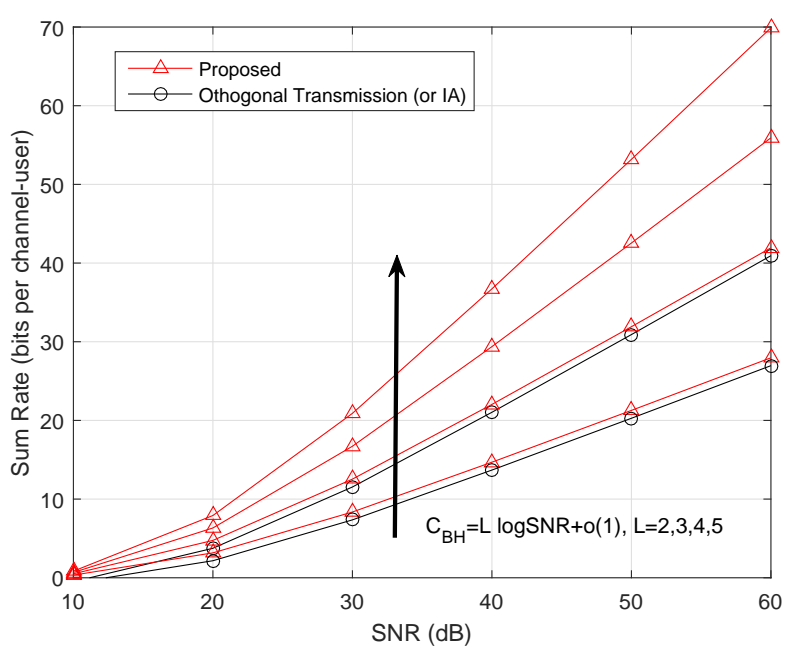

Fig. 4. Achievable sum-rate of the proposed scheme for a DMIMO system with $K=K^{\prime}=2, M=N=3$. Note that a DoF of 6 can be achieved if one of the users is equipped with 4 transmit antennas.

Fig. 5 shows the averaged achievable sum-rates of the proposed scheme for a DMIMO system of $K=K^{\prime}=3, N^{\prime}=N=3$. Here, the BH capacity for the proposed scheme is given by $C_{B H}$ $=L \log \rho+\epsilon, L=2,3,4,5$. The achievable rate of the benchmark scheme using orthogonal transmission and that using IA [21] where $L \geq 3$ are also plotted for comparison. It is demonstrated that the proposed scheme can achieve a considerably greater sumrate over the two benchmark schemes. It is noteworthy that using symbol-extension and considering time-varying channel, the IA scheme can achieve a DoF of 4.5 , which is still less than that achieved by our proposed scheme. Fig. 6 shows the averaged achievable sum-rates of the proposed scheme for a DMIMO system of $K=K^{\prime}=3, N^{\prime}=3, N=6$, where the BH capacity is given by $C_{B H}=L \log \rho+\epsilon, L=2,3,4,5,6,7$. The achievable rate of the benchmark scheme using orthogonal transmission and that using IA where $L \geq 3$ are also plotted for comparison. The proposed scheme can achieve a much greater sum-rate over the orthogonal transmission and IA schemes, due to the higher DoF.

In summary, the propose scheme demonstrates improved performance over the benchmark scheme. Note that in obtaining the above results, no efforts are made to achieve the maximum power gain by optimizing the $\mathrm{NC}$ coefficient matrices and the precoding matrices. This could be considered as an interesting future work. Note that our scheme can also be applied to frequency selective fading channels. For example, the frequency selective fading channel can be first decomposed into flat fading channel using OFDM technique. Then, our proposed signal space alignment based network coding scheme can be applied in a subcarrier-bysubcarrier manner. The achievable rate would be that averaged over all subcarriers, and the result on the degrees of freedom will remain the same as that in the flat fading channel considered in this paper. The DoF result obtained in this way serves as an achievable lower bound on that of a frequency selective fading channel. We note that for an OFDM system with Nc subcarriers, the channel matrix between BS $i$ and User $j$ could be represented by a bigger $N_{i} N_{c}$ by $N_{j}^{\prime} N_{c}$ matrix, by piling up the channel

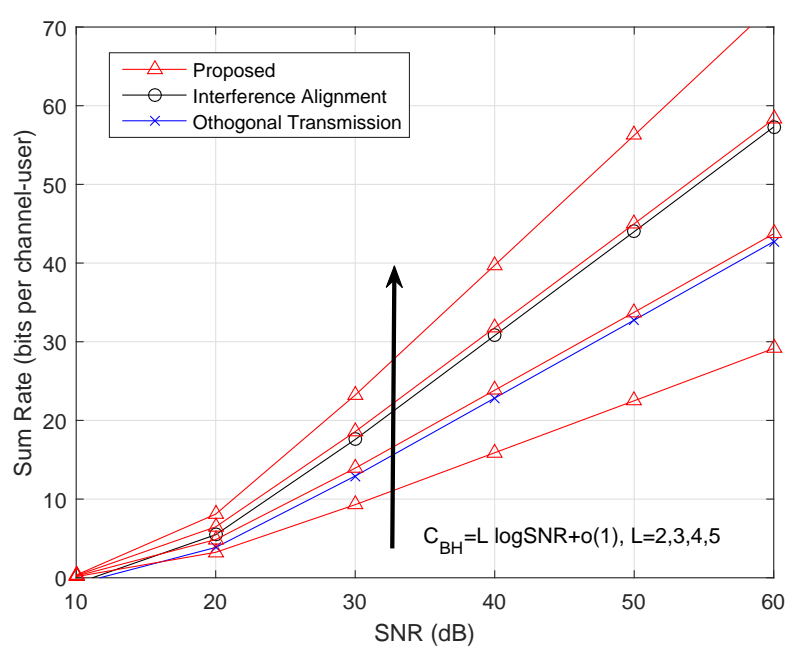

Fig. 5. Achievable sum-rate of the proposed scheme for a DMIMO system with $K=K^{\prime}=3, M=N=3$.

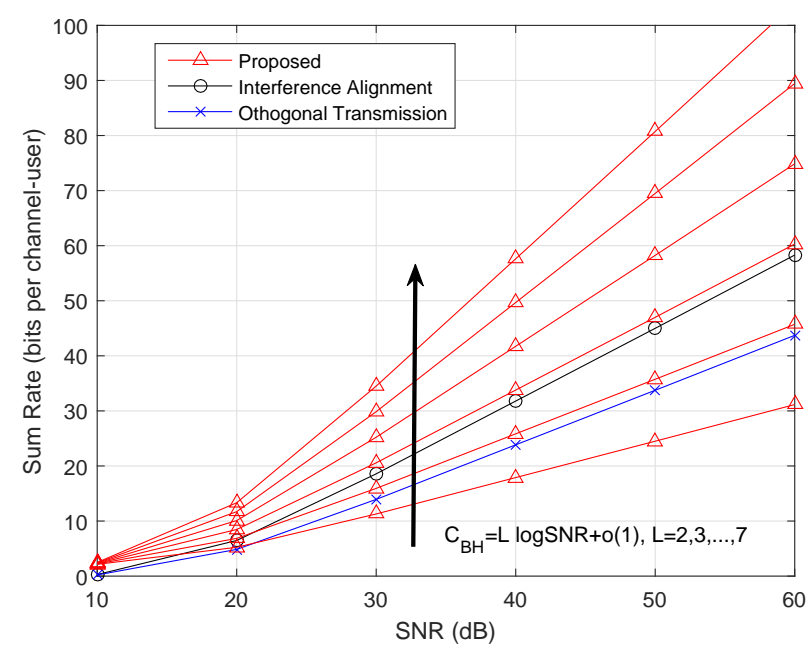

Fig. 6. Achievable sum-rate of the proposed scheme for a DMIMO system with $K=K^{\prime}=3, M=6, N=3$.

matrices of all subcarrier. Therefore, the problem just becomes that under our consideration but with increased dimension. Along this line, the achievable rate analysis applies directly.

\section{CONCLUSIONS AND FUtURE WORKS}

We proposed a new signal-space aligned PNC scheme for DMIMO systems with rate-constrained backhaul. A NC generator matrix is first provided in the proposed scheme, which determines the pattern for signal-space alignment and PNC. Then, precoding matrices are designed to realize the signal-space alignment, providing a platform to conduct PNC to compute the desired NC message sequences. We derived the achievable rate of the proposed scheme and showed that considerably improved degrees of freedom advantage is obtained compared to orthogonal transmission and the interference alignment scheme. In particular, the DoF of the DMIMO with parallel relay is characterized to be $2 \min \left(N, N^{\prime}\right)-1$ if the sum backhaul capacity scales 
like $\left(2 \min \left(N, N^{\prime}\right)-1\right) \log S N R$. This leads to significantly increased DoF compared to that utilizes the strategy for interference channel or orthogonalized transmission, whose DoF is given by $\min \left(N, N^{\prime}\right)$.

This work can be enriched from various aspects. First, it remains unknown whether the different choice of the NC generator matrix $\mathbf{G}$ will have a great impact on the system performance. It is understood that the pattern of signal-space alignment is determined by $\mathbf{G}$, hence a joint optimization of $\mathbf{G}$ and the precoding matrices is expected to yield further performance gain. Second, it remains open whether symbol-extension can further improve the achievable DoF of the proposed scheme, for timevarying channels. Furthermore, how to develop a signal-space aligned PNC scheme for the downlink DMIMO scenario remains as an interesting and important topic. It is believed that these future works deserve further research efforts.

\section{APPENDIX}

Proof of Proposition 1: Denote by A the $N^{\prime} \times N^{\prime}$ matrix $\left[\begin{array}{cc}\mathbf{0} & \alpha \\ \mathbf{I}_{N^{\prime}-1} & \mathbf{0}\end{array}\right]$ over $\mathrm{GF}(q)$, where $\alpha$ is set equal to 2 when $q$ is odd, and is set to a primitive element in $\operatorname{GF}(q)$ otherwise. It can be easily checked that $\operatorname{rank}\left(\mathbf{I}_{N^{\prime}}-\mathbf{A}\right)=N^{\prime}$. Define a $K^{\prime} N^{\prime} \times K^{\prime} N^{\prime}$ matrix $\mathbf{M}_{K^{\prime} N^{\prime}}$ over $\operatorname{GF}(q)$ as

$$
\left[\begin{array}{ccccc}
\mathbf{I}_{N^{\prime}} & \mathbf{I}_{N^{\prime}} & \cdots & & \mathbf{I}_{N^{\prime}} \\
\mathbf{I}_{N^{\prime}} & \mathbf{A} & \mathbf{I}_{N^{\prime}} & \cdots & \mathbf{I}_{N^{\prime}} \\
\mathbf{I}_{N^{\prime}} & \mathbf{I}_{N^{\prime}} & \mathbf{A} & \ddots & \mathbf{I}_{N^{\prime}} \\
& \vdots & & \ddots & \cdots \\
\mathbf{I}_{N^{\prime}} & \cdots & & \mathbf{I}_{N^{\prime}} & \mathbf{A}
\end{array}\right]
$$

By elementary block row operations,

$$
\operatorname{rank}\left(\mathbf{M}_{K^{\prime} N^{\prime}}\right)=\operatorname{rank}\left[\begin{array}{cccc}
\mathbf{I}_{N^{\prime}} & \mathbf{I}_{N^{\prime}} & \cdots & \mathbf{I}_{N^{\prime}} \\
\mathbf{0} & \mathbf{A}-\mathbf{I}_{N^{\prime}} & \mathbf{0} & \ddots \\
\mathbf{0} & \ddots & \ddots & \mathbf{0} \\
\mathbf{0} & \cdots & \mathbf{0} & \mathbf{A}-\mathbf{I}_{N^{\prime}}
\end{array}\right]=K^{\prime} N^{\prime}
$$

Thus, if $L=K^{\prime} N^{\prime}$, then $\mathbf{M}_{K^{\prime} N^{\prime}}$ is a desired matrix over $\operatorname{GF}(q)$ satisfying Properties 1-3.

Consider the case $L=\sum_{j=1}^{K^{\prime}} L_{j}^{\prime}<K^{\prime} N^{\prime}$. Assume that $N^{\prime}=$ $L_{1}^{\prime}=\cdots=L_{k}^{\prime}>L_{k+1}^{\prime} \geq \cdots \geq L_{K^{\prime}}^{\prime}$. As $\left[\begin{array}{cc}\mathbf{M}_{k N^{\prime}} & \mathbf{M}^{\prime} \\ \mathbf{0} & \mathbf{M}^{\prime \prime}\end{array}\right]=$ $k N^{\prime}+\operatorname{rank}(\mathbf{G})$ for any $k N^{\prime} \times\left(L-k N^{\prime}\right)$ matrix $\mathbf{M}^{\prime}$ and $(L-$ $\left.k N^{\prime}\right) \times\left(L-k N^{\prime}\right)$ matrix $\mathbf{G}$, in order to design a generator matrix subject to Properties 1-3 for the case $N^{\prime}=L_{1}^{\prime}=\cdots=$ $L_{k}^{\prime}>L_{k+1}^{\prime} \geq \cdots \geq L_{K^{\prime}}^{\prime}$, it suffices to design a generator matrix subject to Properties 1-3 for the case $N^{\prime}>L_{k+1}^{\prime} \geq \geq \cdots \geq$ $L_{K^{\prime}}^{\prime}$. Thus, without loss of generality, assume $N^{\prime}>\overline{L_{1}^{\prime}} \geq L_{2}^{\prime} \geq$ $\cdots \geq L_{K^{\prime}}^{\prime}$. Form an $L \times L$ matrix $\mathbf{G}$ as follows. Each block $\mathbf{G}_{j k}, 1 \leq j, k \leq K^{\prime}$ is a $L_{j} \times L_{k}$ matrix. $\mathbf{G}_{11}=\mathbf{I}_{L_{1}^{\prime}}$ and $\mathbf{G}_{j j}=\left[\begin{array}{cc}\mathbf{0} & 0 \\ \mathbf{I}_{L_{j}^{\prime}-1} & \mathbf{0}\end{array}\right]$ for $1 \leq j \leq N^{\prime}$. When $1 \leq j<k \leq N^{\prime}$, $\mathbf{G}_{j k}=\left[\begin{array}{c}\mathbf{I}_{L_{j}^{\prime}} \\ \mathbf{0}\end{array}\right]$. When $1 \leq k<j \leq N^{\prime}, \mathbf{G}_{j k}=\left[\begin{array}{ll}\mathbf{I}_{L_{j}^{\prime}} & \mathbf{0}\end{array}\right]$. Property 1 and 2 naturally hold for $\mathbf{G}$. It remains to prove that G has full rank $L$. By re-arranging the $\left(L_{2}^{\prime t h}\right.$ to $L_{1}^{\prime t h}$ rows and columns in $\mathbf{G}$ to the first $L_{1}^{\prime}-L_{2}^{\prime}$ rows and columns, the following matrix can be obtained

$$
\mathbf{G}^{\prime}=\left[\begin{array}{cccccc}
\mathbf{I}_{L_{1}^{\prime}-L_{2}^{\prime}} & & & \mathbf{0} & & \\
& \mathbf{I}_{L_{2}^{\prime}} & \mathbf{I}_{L_{2}^{\prime}} & \mathbf{G}_{23} & \cdots & \mathbf{G}_{2 K^{\prime}} \\
& \mathbf{I}_{L_{2}^{\prime}} & \mathbf{G}_{22} & \mathbf{G}_{23} & \cdots & \mathbf{G}_{2 K^{\prime}} \\
& \mathbf{G}_{32} & \mathbf{G}_{32} & \mathbf{G}_{33} & \cdots & \mathbf{G}_{3 K^{\prime}} \\
& \vdots & \vdots & \vdots & \ddots & \vdots \\
& \mathbf{G}_{K^{\prime} 2} & \mathbf{G}_{K^{\prime} 2} & \mathbf{G}_{K^{\prime} 3} & \cdots & \mathbf{G}_{K^{\prime} K^{\prime}}
\end{array}\right] .
$$

Thus,

$$
\begin{aligned}
\operatorname{rank}(\mathbf{G})= & \operatorname{rank}\left(\mathbf{G}^{\prime}\right)=L_{1}^{\prime}+\operatorname{rank}\left(\mathbf{I}_{L_{2}^{\prime}}+\mathbf{G}_{22}\right) \\
& +\operatorname{rank}\left(\left[\begin{array}{cccc}
\mathbf{I}_{L_{2}^{\prime}} & \mathbf{G}_{23} & \cdots & \mathbf{G}_{2 K^{\prime}} \\
\mathbf{G}_{32} & \mathbf{G}_{33} & \cdots & \mathbf{G}_{3 K^{\prime}} \\
\vdots & \vdots & \ddots & \vdots \\
\mathbf{G}_{K^{\prime} 1} & \mathbf{G}_{K^{\prime} 2} & \cdots & \mathbf{G}_{K^{\prime} K^{\prime}}
\end{array}\right]\right)
\end{aligned}
$$

By similar arguments on the right-most matrix of the above equation, it can be recursively deduced that

$\operatorname{rank}(L)=L_{1}^{\prime}+\operatorname{rank}\left(\mathbf{I}_{L_{2}^{\prime}}+\mathbf{G}_{22}\right)+\cdots+\operatorname{rank}\left(\mathbf{I}_{L_{K}^{\prime}}+\mathbf{G}_{K^{\prime} K^{\prime}}\right)$.

By the definition of $\mathbf{G}_{j j}, \operatorname{rank}\left(\mathbf{I}_{L_{j}^{\prime}}+\mathbf{G}_{j j}\right)=L_{j}$ for all $2 \leq$ $j \leq K^{\prime}$. Thus, $\mathbf{G}=L_{1}^{\prime}+\cdots+L_{K^{\prime}}^{\prime}=L$.

\section{REFERENCES}

[1] D. Lee, H. Seo, B. Clerckx, E. Hardouin, D. Mazzarese, S. Nagata, and K. Sayana, "Coordinated multipoint transmission and reception in lteadvanced: deployment scenarios and operational challenges," IEEE Communications Magazine, vol. 50, no. 2, pp. 148-155, 2012.

[2] J. Zhang, I. Collings, C. Chen, L. Roullet, L. Luo, S. Ho, and J. Yuan, "Evolving small-cell communications towards mobile-over-FTTx networks," IEEE Communications Magazine, 2013.

[3] S. Perlman and A. Forenza, "Distributed-input-distributed-output (DIDO) wireless technology: a new approach to multiuser wireless," Rearden company white paper.

[4] C. Bernardos, A. D. Domenico, J. Ortin, P. Rost, and D. Wübben, "Challenges of designing jointly the backhaul and radio access network in a cloud-based mobile network," in Future Network \& Mobile Summit 2013, Lisbon, Portugal,, Jul 2013.

[5] M. HadZialic, B. Dosenovic, M. Dzaferagic, and J. Musovic, "CloudRAN: Innovative radio access network architecture," ELMAR, 2013 55th International Symposium, pp. 115-120, 2013.

[6] C. Mobile, "C-RAN: the road towards green RAN," White Paper, ver. 2.5, China Mobile Research Institute, Oct. 2011.

[7] Y. Zhou and W. Yu, "Optimized backhaul compression for uplink cloud radio access network," IEEE Jour. Selected Area in Comm., vol. 32, no. 6, pp. 1295-1307, June 2014.

[8] M. G. S. Chia and P. Brick, "The next challenge for cellular networks: backhaul," IEEE Microw. Mag., vol. 10, no. 5, pp. 54-66, Aug. 2009.

[9] S. Z. O. Tipmongkolsilp and A. Jukan, "The evolution of cellular backhaul technologies: current issues and future trends," IEEE Commun. Surveys Tuts., vol. 13, no. 1, pp. 97-113, 2011.

[10] Q. Zhang, C. Yang, and A. Molisch, "Downlink base station cooperative transmission under limited-capacity backhaul," IEEE Trans. Wireless Comm., vol. 12, no. 8, pp. 3746-3759, 2013.

[11] O. Simeone, O. Somekh, H. V. Poor, and S. Shamai, "Local base station cooperation via finite-capacity links for the uplink of linear cellular networks," IEEE Trans. Inf. Theory., vol. 55, no. 1, pp. 190-204, Jan. 2009.

[12] S. Avestimehr, S. Diggavi, and D. Tse, "Wireless network information flow: A deterministic approach," IEEE Trans. Inf. Theory., vol. 57, no. 4, pp. 1872-1905, Apr. 2011.

[13] S. Lim, Y. H. Kim, A. E. Gamal, and S. Chung, "Noisy network coding," IEEE Trans. Inf. Theory., vol. 57, no. 5, pp. 3132-3152, May. 2011.

[14] M. K. Karakayali, G. J. Foschini, and R. A. Valenzuela, "Network coordination for spectrally efficient communications in cellular systems," IEEE Wireless Commun. Mag., vol. 13, no. 4, pp. 56-61, Aug. 2006.

[15] D. Gesbert, S. Hanly, H. Huang, S. Shamai, O. Simeone, and W. Yu, "Multicell MIMO cooperative networks: a new look at interference," IEEE Jour. Sel. Area. Comm., vol. 28, no. 9, pp. 1380-1408, Dec. 2010. 
[16] B. Nazer and M. Gastpar, "Compute-and-forward: Harnessing interference through structured codes," IEEE Trans. Inf. Theory, vol. 57, no. 10, pp. 6463-6486, Oct. 2011

[17] L. Wei and W. Chen, "Network coding in wireless cooperative networks with multiple antenna relays," Proc. International Conf. Wireless Communications and Signal Processing (WCSP), 2012.

[18] S.-N. Hong and G. Caire, "Compute-and-forward strategies for cooperative distributed antenna systems," IEEE Trans. Inf. Theory, vol. 59, no. 9, pp. 5227-5243, Sep. 2013.

[19] T. Yang, X. Yuan, P. Li, I. Collings, and J.Yuan, "A new physicallayer network coding schemewith eigen-direction alignment precoding for MIMO two-way relaying," IEEE Trans. Comm., vol. 61, no. 3, pp. 973-986, Mar. 2013.

[20] J.-B. L. N. Lee and J. Chun, "Degrees of freedom of the MIMO Y channel: signal space alignment for network coding," IEEE Trans. Inf. Theory, vol. 56, no. 7, pp. 3332-3342, July. 2010.

[21] V. Cadambe and S. Jafar, "Interference alignment and the degrees of freedom of the $\mathrm{K}$ user interference channel," IEEE Trans. Inf. Theory, vol. 54, no. 8, pp. 3425-3441, Aug. 2008.

[22] V. Ntranos, V. Cadambe, B. Nazer, and G. Caire, "Integer-forcing interference alignment," IEEE Int. Symp. Inf. Theory, pp. 574-578, 2013.

[23] A. Ghasemi, "Interference alignment for the K user MIMO interference channe," http://arxiv.org/abs/0909.4604, 2009.

[24] M. Maddah-Ali, A. Motahari, and A. Khandani, "Communication over MIMO X channels: Interference alignment, decomposition, and performance analysis," IEEE Trans. Inf. Theory, vol. 54, no. 8, pp. 3457-3470, Aug. 2008.

[25] C. M. Yetis, T. Gou, S. A. Jafar, and A. H. Kayran, "On feasibility of interference alignment in MIMO interference networks," IEEE Trans. Signal Proc., vol. 58, no. 9, pp. 4771-4782, Sept. 2010.

[26] O. Simeone, "Distributed MIMO in multi-cell wireless systems via finitecapacity links," in Proc. ISCCSP, 2008.

[27] P. Marsch, "Coordinated multi-point under a constrained backhaul and imperfect channel knowledge," VOGT, 2010.

[28] T. Yang, Q. T. Sun, A. Zhang, and J.Yuan, "A linear network coding approach for uplink distributed MIMO systems : protocol and outage behavior," IEEE Journal on Selected Areas Comm., vol. 33, no. 2, pp. 250263, Feb. 2015.

[29] T. M. Cover and J. A. Thomas, "Elements of information theory," John Wiley \& Sons, Inc., 1991.

[30] W. Nam, S. Chung, and Y. H. Lee, "Capacity of the gaussian two-way relay channel to within 1/2 bit," IEEE Trans. Inf. Theory, vol. 56, no. 11, pp. 5488-5494, Nov. 2010.

[31] T. Huang, T. Yang, J. Yuan, and I. Land, "Design of irregular repeataccumulate coded physical-layer network coding for gaussian two-way relay channels," IEEE Trans. Comm., vol. 61, no. 3, pp. 897-909, Mar. 2013.

[32] L. Yang, T. Yang, J.Yuan, and P. An, "Achieving the near-capacity of twoway relay channels with modulation-coded physical-layer network coding," IEEE Trans. Wireless Comm., vol. 14, no. 9, pp. 5225-5239, May 2015.

[33] J. Zhan, B. Nazer, U. Erez, and M. Gastpar, "Integer-forcing linear receivers," IEEE Trans. Inf. Theory, vol. 60, no. 12, pp. 7661-7685, Dec. 2014.

[34] S. Hong and G. Caire, "Structured lattice codes for some two-user gaussian networks with cognition, coordination, and two hops," IEEE Trans. Inf. Theory, vol. 61, pp. 2624-2646, May 2015.

[35] S. Zhang, S. Liew, and P. P. Lam, "Hot topic: physical-layer network coding," Proceedings of the 12th annual international conference on Mobile computing and networking, pp. 358-365, 2006.

[36] T. Yang, "Distributed MIMO broadcasting: signal-space alignment and reverse compute-and-forward," submitted to IEEE Trans. Wireless Comm., 2016. 